\title{
Medicinal plants with cholesterol-lowering effect marketed in the Buenos Aires-La Plata conurbation, Argentina: An Urban Ethnobotany study
}

\author{
Julio Alberto Hurrell*, Jeremías P. Puentes, and Patricia M. Arenas
}

\begin{abstract}
This contribution presents 82 species of medicinal plants whose products are sold and consumed as cholesterollowering in the Buenos Aires-La Plata conurbation, Argentina. The hypocholesterolemic effect is relevant because the high level of blood cholesterol is one of the major risk factors for cardiovascular diseases, leading causes of death and disability almost worldwide. The species were selected from their locally assigned use, obtained from interviews with informants, data labels, prospectus and leaflets of products, and information diffused in the media, specially the Internet. Furthermore, a bibliographic review on scientific studies that validate the biological activity and effects of each species was accomplished. The theoretical framework of this research argues that urban botanical knowledge comprising some knowledge linked to traditions of various immigrants segments (invisible to most of the local population), and other non-traditiona/knowledge (visible). The assessment of these kinds of knowledge is approached from the plant products circulation in the restricted circuits of immigrants (Bolivian and Chinese for this contribution) and the general commercial circuit, so that products that pass from the first circuit to the second gain visibility. The circulation of plant products that acquire visibility also expressed the botanical knowledge transmission from one context to another, a transmission enhanced and accelerated by the media. In this framework, the visibility levels of plant products considered hypocholesterolemic in the study area are discussed.
\end{abstract}

Keywords: Multicultural contexts, methodological approach, plants and knowledge visibility, botanical knowledge transmission

\section{INTRODUCTION}

This contribution is framed in a research line in Urban Ethnobotany of the Laboratorio de Etnobotánica y Botánica Aplicada (LEBA). This research line argues that botanical knowledge (BK) in multicultural urban contexts is a set of knowledge and beliefs about different plant elements of the environment (plants, parts parts thereof, and derived products). This urban BK contains two qualities of knowledge that interact in several ways: 1) non-traditional (taught and disseminated knowledge, including the scientific knowledge), and 2) linked to traditions (the origin traditions of various immigrant groups, local longstanding family traditions). Urban BK orients selection and use strategies of plant products, which are evidenced in the dynamics of their diffusion inside the commercial circuits. Thus, in assessing the circulation of products is possible to rebuild the BK that orients those

1 Laboratorio de Etnobotánica y Botánica Aplicada (LEBA), Facultad de Ciencias Naturales y Museo, Universidad Nacional de La Plata. Calle 64 nro. 3, 1900-La Plata. Consejo Nacional de Investigaciones Científicas y Técnicas (CONICET), República Argentina.

* Corresponding author. E-mail address:juliohurrell@gmail.com 
strategies. This is a methodological proposal that the research line of the LEBA has been developing for over a decade in the Buenos Aires-La Plata conurbation, Argentina (Hurrell 2014; Hurrell and Pochettino 2014).

Immigrants from different origin and residence time incorporate to the urban areas both new plant elements as the knowledge about these. Some of the plant elements persist inside the restricted commercial circuit of each group of immigrants, and remain invisible to the majority of local population (knowledge linked to traditions). Other plant elements enter to the general commercial circuit and become visible (non-traditional knowledge). The passage from the restricted circuit of immigrants to the general commercial circuit is considered a visualization process in which the uses of plants change their meaning according to the change of context: the meaning of some uses change, new meanings are added (Hurrell 2014). In the visualization process different agents are involved, such as natural products stores locally called dietéticas (specialized in selling healthy foods, nutraceuticals, phytotherapics, dietary supplements), and also the mass media (mainly the Internet), which enhance the BK transmission rapidly and in multiple directions (Pochettino and Hurrell 2013; Hurrell et al. 2015).

This paper focuses on medicinal plants products consumed as cholesterol-lowering agents in the study area. This selection is based on the wide dissemination of information (knowledge), especially through the media, in relation to reducing the risk of cardiovascular diseases that involves the use of hypocholesterolemic agents. The relevance of these agents in urban areas is reflected in the wide variety of plant products marketed for that purpose.

Hypercholesterolemia is an increase in the normal concentration of cholesterol in the blood, and is one of the major risk factors for cardiovascular diseases such as atherosclerosis, stroke, and myocardial infarction, leading causes of death and disability almost worldwide (Deng 2009). Maintaining cholesterol homeostasis involves various regulatory mechanisms associated with its synthesis, absorption, metabolism, elimination. Hypercholesterolemia is a mismatch metabolic resulting from those processes. The hypocholesterolemic agents reduce the high cholesterol levels, hence, the cardiovascular risk (Maza Cave et al. 2000).

Hypercholesterolemia is a type of hyperlipidemia, ie, high levels of blood lipids, including triglycerides, cholesterol, and lipoproteins that make possible the cholesterol transport in blood plasma. Cholesterollipoprotein complexes with very low, low, and intermediate densities are named proatherogenic cholesterol and are cardiovascular risk factors. In contrast, cholesterol-lipoprotein complex with high density is named antiatherogenic cholesterol and has protective effect on cardiovascular disease (Deng 2009). The hypolipidemic agents reduce the risk of cardiovascular diseases by lowering total cholesterol, pro-atherogenic cholesterol, and triglycerides levels (García Mesa 2014).

Hyperlipidemia and hypolipidemia (its opposite) are types of dyslipidemias. The generic term dyslipidemia refers to alterations in the synthesis, transport or metabolism of lipids, which modify the plasma concentrations of total cholesterol, transporter lipoproteins, and triglycerides (Furgione et al. 2009; García Mesa 2014). Primary dyslipidemia is due to genetic factors, and secondary dyslipidemia is due to environmental factors (diet, sedentary lifestyle) or pathologies such as obesity, diabetes, metabolic syndrome, among others (Maza Cave et al. 2000; Alegría Ezquerra et al. 2008).

The aim of this contribution is to present 82 species of medicinal vascular plants whose products are commercialized and consumed as cholesterol-lowering inside the Buenos AiresLa Plata conurbation. Also, regarding to the methodological proposal of the research line, show that hypocholesterolemic plant products illustrate the dynamics of urban BK, starting from the dissemination of the different products in the local commercial circuits. 


\section{MATERIALS AND METHODS}

The study area corresponds to the Buenos Aires-La Plata conurbation, which comprises two contiguous urban agglomerates: Greater Buenos Aires and Greater La Plata. The first includes the Ciudad Autónoma de Buenos Aires, the capital of Argentina, and the neighboring districts of the Buenos Aires province. According to the national census of 2010, the Greater Buenos Aires had about $13,000,000$ inhabitants in 3850 square kilometers. In Buenos Aires city live 3,000,000 people at only 203 square kilometers. This widespread urban agglomerate is the largest metropolitan area in size and population of the country, the second in South America, the third in Latin America, the fifth in America and the seventeenth in the world.

Greater La Plata includes the city of La Plata, the capital of Buenos Aires province, located in the homonymous district, and the neighboring districts of Ensenada and Berisso. According to the above mentioned census this urban agglomerate had about 800,000 inhabitants in 1150 square kilometers. The conurbation has a total area of 5,000 square kilometers, in which live about $13,800,000$ inhabitants (Puentes and Hurrell 2015).

The research methodological approach is strictly qualitative, based on usual ethnobotanical techniques: participant observation, free listings, free and semistructured interviews, applied according to the specific comments and suggestions of differents authors (Martin 1995; BlancoCastro 1996; Quinlan 2005; Stepp 2005; Etkin and Ticktin 2010; Albuquerque et al. 2014). In particular, semi-structured interview questions were focused to identify the hypocholesterolemic use of plant products, and other related, as well as its diffusion level.

Data collection is performed without interruption since 2005, in 150 of outlets: 115 shops of the general commercial circuit (dietéticas, herbal stores) and 35 outlets of the restricted circuits of two groups of immigrants: Chinese and Bolivian, in the
Ciudad Autónoma de Buenos Aires. For the Chinese segment was relieved five supermarkets of the called Barrio Chino, a sector of Belgrano neighborhood, that sell products of the Traditional Chinese Herbal Medicine, imported from China (Hurrell and Pochettino 2014). For the Bolivian segment was relieved 30 outlets, the total shops and stalls that conform the Bolivian market in Liniers neighborhood, an example of traditional market nestled in an urban context (Pochettino et al. 2012; Puentes and Hurrell 2015). The selection of outlets of general commercial circuit started randomly and stopped upon reaching saturation of information about the products surveyed.

Two informants for each outlet were selected, so 300 people were interviewed previous informed consent. About $80 \%$ of those interviewed are sellers of both sexes and different ages, who know the properties and benefits of the products that they sell, and guide the consumers on their forms of employment and administration. The informants of the Chinese and Bolivian immigrants segments are mostly originally from their respective countries. The informants of the general commercial circuit are Argentine.

Samples of the products of each species were obtained in all outlets. Each sample was designated with an alphanumeric code and was deposited in the ethnobotanical collections of the LEBA. Commercial products - fragmented plant material, tinctures, dietary supplements (tablets, capsules), among others - indicate their components in their respective labels. However, materials not labeled, or with doubts about its composition, were identified by morphological characters and micrographic analysis (Vignale and Gurni 2007).

The medicinal species surveyed respond to the locally assigned use: "cholesterollowering" or "hypocholesterolemic" obtained from the interviews, data labels, prospectus and leaflets of each product, and the information disseminated in graphic media and the Internet. All data were confronted with the available ethnobotanical literature. 
The locally assigned use is constructed from the urban BK (linked to traditions and nontraditional), and from changes of meaning that happen in the dynamics of the transmission of local BK (Hurrell 2014).

Also, a bibliographic review about diverse academic studies of effects and biological activity of each species was accomplished. These studies validate the locally assigned use in the scientific context. Certainly, each species has other medicinal uses, in some cases numerous, but have been omitted for reasons of space. Regarding academic studies analyzed was selected the hypocholesterolemic effect as well as others linked effects, as hypolipidemic, antidyslipidemic, anti-atherogenic.

The cholesterol-lowering effect can be obtained from an appropriate diet, which includes certain food plants such as soybeans, Glycine max (L.) Merr. (Mateos-Aparicio et al. 2008), chickpeas, Cicer arietinum L. (Jukanti et al. 2012), onions, Allium cepa L. (García Mesa 2014), among others. The hypocholesterolemic food plants were not considered in this contribution, which only includes plant species selected and consumed for medicinal purposes. However, there are products of species clearly identified as foods such as leaves and roots of chicory, Cichorium intybus L., or fruits of mango, Mangifera indica $L$, that were included because they have another products that are consumed as therapeutics: fragmented aerial parts of chicory, fragmented leaves of mango. In this sense it is important to highlight that the selection of the species in this study also relies on the plant products circulating in the commercial circuit marketed for medicinal purposes.

Moreover, several species of aromatic plants were included because are used as therapeutics and at the same time as food condiments and/or beverages flavoring. In all the cases, the aromatic species treated here were identified as therapeutics in the interviews, eg, products of ginger, Zingiber officinale Roscoe, and turmenic, Curcuma longa L. (fragmented and powdered rhizomes), but products of cumin, Cuminum cyminum L. (mericarps) and rosemary, Rosmarinus officinalis L. (fragmented leaves), among others, were excluded because are only considered as condiment, despite that their hypocholesterolemic effect has been investigated (Andallu and Ramya 2007; Afonso et al. 2013).

\section{RESULTS}

Table 1 includes the results obtained for the 82 species surveyed: scientific names, families, vernacular names, different types of products, samples, and references about biological activity and effects studied. The exclusive plant products from Bolivian market are indicated with an asterisk $(*)$, and the exclusive products from supermarkets in Barrio Chino are indicated with two asterisks $(* *)$. Products without asterisk belong to general commercial circuit. The products sold correspond to dried materials (or derivatives like tinctures, solid or liquid extracts) except those indicated as fresh (eg, fresh plants, fresh fruits). 
Table 1. Medicinal plants with cholesterol-lowering effect commercialized in the Buenos Aires-La Plata conurbation, Argentina.

\begin{tabular}{|c|c|c|}
\hline $\begin{array}{l}\text { Species, families, } \\
\text { local names }\end{array}$ & Products types [Samples] & Biological activity and effects studied \\
\hline $\begin{array}{l}\text { Achillea millefolium } \mathrm{L} . \\
\text { Asteraceae } \\
\text { Milenrama, aquilea }\end{array}$ & $\begin{array}{l}\text { Fragmented aerial parts } \\
\text { [C001] } \\
\text { Tincture }[\mathrm{H} 348]\end{array}$ & $\begin{array}{l}\text { Hypocholesterolemic, hypolipidemic } \\
\text { (Mustafa et al. 2012; Hurrell and Puentes } \\
\text { 2013). }\end{array}$ \\
\hline $\begin{array}{l}\text { Achyrocline satureioides } \\
\text { (Lam.) DC. } \\
\text { Asteraceae } \\
\text { Marcela }\end{array}$ & $\begin{array}{l}\text { Fragmented aerial parts } \\
{[\mathrm{H} 097]^{*}[\mathrm{H} 448]} \\
\text { Herbal tea in bags (mixture) } \\
{[\mathrm{H} 412]} \\
\text { Tincture }[\mathrm{H} 345]\end{array}$ & $\begin{array}{l}\text { Hypocholesterolemic, hypolipidemic (Espiña } \\
\text { et al. 2012). }\end{array}$ \\
\hline $\begin{array}{l}\text { Acorus calamus } \mathrm{L} \text {. } \\
\text { Acoraceae } \\
\text { Cálamo aromático }\end{array}$ & Fragmented rhizomes [P173] & $\begin{array}{l}\text { Hypocholesterolemic, hypolipidemic, anti- } \\
\text { dyslipidemic (Parab and Menghi 2002; } \\
\text { Divya et al. 2011; Shafi and Tabassum 2013). }\end{array}$ \\
\hline $\begin{array}{l}\text { Allium ampeloprasum } \mathrm{L} \text {. } \\
\text { Amaryllidaceae } \\
\text { Ajo macho }\end{array}$ & Bulbs [P255]*[F013] [P294] & $\begin{array}{l}\text { Hypocholesterolemic, hypolipidemic } \\
\text { (Roghani and Aghaie 2007; Dey and Khaled } \\
\text { 2013; Puentes \& Hurrell 2015). }\end{array}$ \\
\hline $\begin{array}{l}\text { Allium sativum L. } \\
\text { Amaryllidaceae } \\
\text { Ajo }\end{array}$ & Tablets [H329] & $\begin{array}{l}\text { Hypocholesterolemic,hypolipidemic,anti- } \\
\text { dyslipidemic, anti-atherosclerotic (Bordia et } \\
\text { al. 1998;Koscielny et al. 1999; García Mesa } \\
\text { 2014). }\end{array}$ \\
\hline $\begin{array}{l}\text { Aloysia citriodora Palau } \\
\text { Verbenaceae } \\
\text { Cedrón }\end{array}$ & $\begin{array}{l}\text { Fragmented leaves [C009] } \\
\text { Herbal tea in bags [H044] } \\
\text { Herbal tea in bags (mixture) } \\
\text { [H047] }\end{array}$ & No data. \\
\hline $\begin{array}{l}\text { Alpinia officinarum Hance } \\
\text { Zingiberaceae } \\
\text { Galanga }\end{array}$ & $\begin{array}{l}\text { Fragmented rhizomes [P176] } \\
\text { Powdered rhizomes }[\mathrm{C} 120]\end{array}$ & $\begin{array}{l}\text { Hypocholesterolemic, hypolipidemic, anti- } \\
\text { lipogenic (Shin et al. 2003; Xia et al. 2010; } \\
\text { Jung et al. 2012). }\end{array}$ \\
\hline $\begin{array}{l}\text { Amorphophallus konjac } \\
\text { K. Koch } \\
\text { Araceae } \\
\text { Konjac }\end{array}$ & Tablets [SD29] [SD32] & $\begin{array}{l}\text { Hypocholesterolemic, hypolipidemic, anti- } \\
\text { atherosclerotic (Chen et al. 2003b; Vasques } \\
\text { et al. 2008; Yanai et al. 2015). }\end{array}$ \\
\hline $\begin{array}{l}\text { Angelica archangelica L. } \\
\text { Apiaceae } \\
\text { Angélica }\end{array}$ & Fragmented roots [C006] & $\begin{array}{l}\text { Hypocholesterolemic, hypolipidemic (Alabi } \\
\text { etal. 2013). }\end{array}$ \\
\hline $\begin{array}{l}\text { Angelica sinensis (Oliver) } \\
\text { Diels } \\
\text { Apiaceae } \\
\text { Angélica china }\end{array}$ & Fragmented roots $[\mathrm{H} 399]^{* *}$ & $\begin{array}{l}\text { Hypocholesterolemic, hypolipidemic, anti- } \\
\text { atherosclerotic ( Li et al. 2000; Wu and Hsieh } \\
\text { 2011; Wang et al. 2015). }\end{array}$ \\
\hline $\begin{array}{l}\text { Artemisia absinthium L. } \\
\text { Asteraceae } \\
\text { Ajenjo }\end{array}$ & $\begin{array}{l}\text { Fresh plants }[\mathrm{H} 165]^{*} \\
\text { Fragmented aerial parts } \\
{[\mathrm{H} 451]} \\
\text { Tincture }[\mathrm{H} 356]\end{array}$ & $\begin{array}{l}\text { Hypocholesterolemic, hypolipidemic, anti- } \\
\text { atherosclerotic (Alabi et al. 2013; Daradka et } \\
\text { al. 2014). }\end{array}$ \\
\hline $\begin{array}{l}\text { Aspidosperma quebracho- } \\
\text { blanco Schltdl. } \\
\text { Apocynaceae } \\
\text { Quebracho }\end{array}$ & Fragmented bark [H219] & No data. \\
\hline $\begin{array}{l}\text { Astragalus mongholicus } \\
\text { Bunge [= A. membranaceus } \\
\text { Bunge] } \\
\text { Leguminosae } \\
\text { Astrágalo }\end{array}$ & $\begin{array}{l}\text { Roots }[\mathrm{H} 400]^{* *} \\
\text { Fragmented roots [P183] } \\
\text { Capsules (mixture) [H323] }\end{array}$ & $\begin{array}{l}\text { Hypocholesterolemic, hypolipidemic, anti- } \\
\text { atherosclerotic (Li et al. 2000; Ma et al. 2011; } \\
\text { Wang et al. 2012). }\end{array}$ \\
\hline
\end{tabular}




\begin{tabular}{|c|c|c|}
\hline $\begin{array}{l}\text { Species, families, } \\
\text { local names }\end{array}$ & Products types [Samples] & Biological activity and effects studied \\
\hline $\begin{array}{l}\text { Atractylodes lancea } \\
\text { (Thunb.) DC. [= Atractylis } \\
\text { ovataThunb.] } \\
\text { Asteraceae } \\
\text { Cang zhu }\end{array}$ & $\begin{array}{l}\text { Fragmented rhizomes } \\
{[\mathrm{H} 446]^{* *}}\end{array}$ & Hypolipidemic (Han et al. 2009). \\
\hline $\begin{array}{l}\text { Baccharis articulata (Lam.) } \\
\text { Pers. } \\
\text { Asteraceae } \\
\text { Carqueja }\end{array}$ & $\begin{array}{l}\text { Fresh plants [B416]* } \\
\text { Fragmented aerial parts } \\
\text { [P143]* [H449] }^{*} \text { Herbal tea in bags [H411] } \\
\text { Tincture [H346] } \\
\text { Tablets (mixture) [H501] }\end{array}$ & No data. \\
\hline $\begin{array}{l}\text { Baccharis trímera (Less.) } \\
\text { DC. } \\
\text { AsTERACEAE } \\
\text { Carqueja }\end{array}$ & $\begin{array}{l}\text { Fresh plants [B424]* } \\
\text { Fragmented aerial parts } \\
{[\text { C017] }}\end{array}$ & $\begin{array}{l}\text { Hypocholesterolemic, hypolipidemic, anti- } \\
\text { dyslipidemic (Pizziolo et al. 2011; Souza et al. } \\
\text { 2011, 2012). }\end{array}$ \\
\hline $\begin{array}{l}\text { Bauhinia forficata Link } \\
\text { subsp. pruinosa (Vogel) } \\
\text { Fortunato \&Wunderlin } \\
\text { Leguminosae } \\
\text { Pata de vaca }\end{array}$ & $\begin{array}{l}\text { Fragmented leaves [H015] } \\
\text { [H049] }\end{array}$ & $\begin{array}{l}\text { Hypocholesterolemic, hypolipidemic (Lino } \\
\text { et al. 2004; Barboza et al. 2009; Pizziolo et al. } \\
\text { 2011). }\end{array}$ \\
\hline $\begin{array}{l}\text { Bixa orellana } L . \\
\text { Bixaceae } \\
\text { Achiote, urucú }\end{array}$ & Seeds $[\mathrm{H} 398]^{*}[\mathrm{H} 283]$ & $\begin{array}{l}\text { Hypocholesterolemic, hypolipidemic, anti- } \\
\text { atherosclerotic (Paula et al. 2009; Ferreira et } \\
\text { al. 2013; García Mesa 2014). }\end{array}$ \\
\hline $\begin{array}{l}\text { Calendula officinalis L. } \\
\text { Asteraceae } \\
\text { Caléndula }\end{array}$ & $\begin{array}{l}\text { Inflorescences [H200] } \\
\text { Tincture }[\mathrm{H} 341]\end{array}$ & $\begin{array}{l}\text { Hipocolesterolémico, hipolipidémico, } \\
\text { anti-atherogenic (Lastra Valdés and Piquet } \\
\text { García 1999; Liu and Guo 2010; Orekhov } \\
\text { 2013). }\end{array}$ \\
\hline $\begin{array}{l}\text { Cassia fistula L. } \\
\text { Leguminosae } \\
\text { Cañafístula }\end{array}$ & Legumes [H228] & $\begin{array}{l}\text { Hypocholesterolemic, hypolipidemic, } \\
\text { anti-dyslipidemic (El-Saadany et al. 1991; } \\
\text { Nirmala et al. 2008; Singh et al. 2010). }\end{array}$ \\
\hline $\begin{array}{l}\text { Celidonium majus L. } \\
\text { Papaveraceae } \\
\text { Celidonia }\end{array}$ & $\begin{array}{l}\text { Fragmented aerial parts } \\
{[\mathrm{H} 120][\mathrm{H} 179]}\end{array}$ & $\begin{array}{l}\text { Hipocolesterolémico, hipolipidémico (Zarei } \\
\text { et al. 2014). }\end{array}$ \\
\hline $\begin{array}{l}\text { Centella asiatica (L.) Urb. } \\
\text { Apiaceae } \\
\text { Centella }\end{array}$ & $\begin{array}{l}\text { Fragmented aerial parts } \\
\text { [H076]* } \\
\text { Tincture [H321] } \\
\text { Tablets [H403] } \\
\text { Tablets (mixture) [H404] } \\
\text { Capsules [H380] } \\
\text { Capsules (mixture) [H405] }\end{array}$ & $\begin{array}{l}\text { Hipocolesterolémico, hipolipidémico, lipid } \\
\text { modulator (Pingale 2008; Hussin et al. 2009; } \\
\text { Chauhan et al. 2010). }\end{array}$ \\
\hline $\begin{array}{l}\text { Cichoriun intybus L. } \\
\text { Asteraceae } \\
\text { Achicoria }\end{array}$ & $\begin{array}{l}\text { Fragmented aerial parts } \\
{[\mathrm{H} 129]}\end{array}$ & $\begin{array}{l}\text { Hypocholesterolemic, hypolipidemic, anti- } \\
\text { dyslipidemic (Kocsis et al. 2003; Shafi and } \\
\text { Tabassum 2013). }\end{array}$ \\
\hline $\begin{array}{l}\text { Cola nítida (Vent.) Schott } \\
\text { \& Endl. } \\
\text { Malvaceae } \\
\text { Cola, nuez de cola }\end{array}$ & Tincture [H295] & $\begin{array}{l}\text { Hypocholesterolemic, hypolipidemic (Nku et } \\
\text { al. 2014; Dah-Nouvlessounon et al. 2015). }\end{array}$ \\
\hline $\begin{array}{l}\text { Crataegus laevigata (Poir.) } \\
\text { DC. } \\
\text { Rosaceae } \\
\text { Crataegus }\end{array}$ & Fragmented leaves [H153] & $\begin{array}{l}\text { Hypocholesterolemic, hypolipidemic (Dalli } \\
\text { et al. 2011; Robert et al. 2012; Littleton et al. } \\
\text { 2013). }\end{array}$ \\
\hline $\begin{array}{l}\text { Curcuma longa L. } \\
\text { Zingiberaceae } \\
\text { Cúrcuma }\end{array}$ & $\begin{array}{l}\text { Fragmented rhizomes [C136] } \\
\text { Powdered rhizomes [C036] }\end{array}$ & $\begin{array}{l}\text { Hypocholesterolemic, hypolipidemic, anti- } \\
\text { atherosclerotic (Mesa et al. 2000; Megraj et } \\
\text { al. 2011; Ibrahim et al. 2013; Saravanan and } \\
\text { Ignacimuthu 2015). }\end{array}$ \\
\hline
\end{tabular}




\begin{tabular}{|c|c|c|}
\hline $\begin{array}{l}\text { Species, families, } \\
\text { local names }\end{array}$ & Products types [Samples] & Biological activity and effects studied \\
\hline $\begin{array}{l}\text { Cymbopogon citratus (DC.) } \\
\text { Stapf } \\
\text { Poaceae } \\
\text { Pasto limón }\end{array}$ & $\begin{array}{l}\text { Fresh tillers [FB407]* }{ }^{*} \text { RF08] } \\
\text { Fragmented tillers }[\mathrm{H} 171]\end{array}$ & $\begin{array}{l}\text { Hypocholesterolemic, hypolipidemic, anti- } \\
\text { dyslipidemic, anti-atherogenic (Orrego et } \\
\text { al. 2009; Bidkar et al. 2011; Costa et al. 2011; } \\
\text { Souza et al. 2012; Ekpenyong et al. 2014). }\end{array}$ \\
\hline $\begin{array}{l}\text { Cynara cardunculus L. } \\
\text { [= C. scolymus L.] } \\
\text { Asteraceae } \\
\text { Alcachofa }\end{array}$ & $\begin{array}{l}\text { Fragmented leaves [H069] } \\
\text { Tincture [H333] } \\
\text { Herbal tea in bags [H093]* } \\
\text { [H411] } \\
\text { Tablets [H094]* } \\
\text { Tablets (mixture) [H501] }\end{array}$ & $\begin{array}{l}\text { Hypocholesterolemic, hypolipidemic, anti- } \\
\text { dyslipidemic, anti-atherosclerotic (Brown } \\
\text { and Rice-Evans 1998; Souza et al. 2012; } \\
\text { lbrahim et al. 2013; Wider et al. 2013; Falé et } \\
\text { al. 2014). }\end{array}$ \\
\hline $\begin{array}{l}\text { Eucommia ulmoides Oliv. } \\
\text { Eucommiaceae } \\
\text { Du zhong }\end{array}$ & Fragmented bark $[\mathrm{H} 447]^{* *}$ & $\begin{array}{l}\text { Hypocholesterolemic, hypolipidemic, anti- } \\
\text { atherosclerotic(Yen and Hsieh 1998; Choi et } \\
\text { al. 2008; He et al. 2014). }\end{array}$ \\
\hline $\begin{array}{l}\text { Eugenia uniflora L. } \\
\text { Myrtaceae } \\
\text { Pitanga }\end{array}$ & Fragmented leaves [H140] & $\begin{array}{l}\text { Hypocholesterolemic, hypolipidemic,anti- } \\
\text { dyslipidemic (Bongiolo 2008; Barboza et al. } \\
\text { 2009; Pizziolo et al. 2011). }\end{array}$ \\
\hline $\begin{array}{l}\text { Euterpe oleracea Mart. } \\
\text { Arecaceae } \\
\text { Açaí }\end{array}$ & Capsules [H302] [H500] & $\begin{array}{l}\text { Hypocholesterolemic, hypolipidemic, anti- } \\
\text { atherosclerotic (Devalaraja et al. 2011; Souza } \\
\text { et al. 2010, 2012). }\end{array}$ \\
\hline $\begin{array}{l}\text { Foeniculum vulgare Mill. } \\
\text { Apiaceae } \\
\text { Hinojo }\end{array}$ & $\begin{array}{l}\text { Mericarps [C047] } \\
\text { Powdered mericarps [C121] } \\
\text { Tincture [H274] }\end{array}$ & $\begin{array}{l}\text { Hypocholesterolemic, hypolipidemic, } \\
\text { anti-atherogenic (Badgujar et al. 2014; } \\
\text { Oulmouden et al. 2014). }\end{array}$ \\
\hline $\begin{array}{l}\text { Garcinia gummi-gutta Roxb. } \\
\text { [= G. cambogia (Gaertn.) } \\
\text { Desr.] } \\
\text { Clusiaceae } \\
\text { Garcinia }\end{array}$ & $\begin{array}{l}\text { Fruits [H305]** } \\
\text { Tincture [H303] } \\
\text { Tablets [H318] [SD41] } \\
\text { Capsules (mixture) [H405] }\end{array}$ & $\begin{array}{l}\text { Hypocholesterolemic, hypolipidemic, anti- } \\
\text { lipogenic, anti-atherogenic (Koshy et al. } \\
\text { 2001; Vasques et al. 2008; Bidkar et al. 2011; } \\
\text { Ateş et al. 2012; Semwal et al. 2015). }\end{array}$ \\
\hline $\begin{array}{l}\text { Gentianella alborosea } \\
\text { (Gilg.) Fabris } \\
\text { Gentianaceae } \\
\text { Hercampuri }\end{array}$ & $\begin{array}{l}\text { Fragmented aerial parts } \\
\text { [P155] } \\
\text { Capsules [H377] [P277] }\end{array}$ & $\begin{array}{l}\text { Hypolipidemic (Li et al. 2010; Puentes and } \\
\text { Hurrell 2015). }\end{array}$ \\
\hline $\begin{array}{l}\text { Ginkgo biloba L. } \\
\text { Ginkgoaceae } \\
\text { Ginkgo }\end{array}$ & $\begin{array}{l}\text { Fragmented leaves [H052] } \\
\text { Tablets [H111] [H327] } \\
\text { Capsules (mixture) [H323] }\end{array}$ & $\begin{array}{l}\text { Hypocholesterolemic, hypolipidemic, anti- } \\
\text { dyslipidemic, anti-atherosclerotic (Yao et al. } \\
\text { 2007; Xie et al. 2009; Wei et al. 2013). }\end{array}$ \\
\hline $\begin{array}{l}\text { Glycyrrhiza glabra L. } \\
\text { Leguminosae } \\
\text { Regaliz }\end{array}$ & $\begin{array}{l}\text { Fragmented roots [H050] } \\
\text { Powdered roots [H035] } \\
\text { Herbal tea in bags (mixture) } \\
\text { [H046] } \\
\text { Capsules (mixture) [H323] }\end{array}$ & $\begin{array}{l}\text { Hypocholesterolemic, hypolipidemic,anti- } \\
\text { dyslipidemic,anti-atherosclerotic (Asgary } \\
\text { et al. 2007; Maurya et al. 2009; Megraj et } \\
\text { al. 2011; Dhaliya et al. 2013; Saravanan and } \\
\text { Ignacimuthu 2015). }\end{array}$ \\
\hline $\begin{array}{l}\text { Handroanthus } \\
\text { impetiginosus (Mart. ex } \\
\text { DC.) Mattos } \\
\text { [=Tabebuia impetiginosa } \\
\text { (Mart. ex DC.) Standl.] } \\
\text { Bignoniaceae } \\
\text { Lapacho }\end{array}$ & Fragmented bark [H207] & $\begin{array}{l}\text { Hypocholesterolemic, hypolipidemic, anti- } \\
\text { atherosclerotic (Pizziolo et al. 2011; Kiage- } \\
\text { Mokua et al. 2012; Choi et al. 2014). }\end{array}$ \\
\hline $\begin{array}{l}\text { Heimia salicifolia (Kunth) } \\
\text { Link } \\
\text { Lythraceae } \\
\text { Quiebra arado }\end{array}$ & $\begin{array}{l}\text { Fragmented aerial parts } \\
\text { [H124] }\end{array}$ & No data. \\
\hline $\begin{array}{l}\text { Hibiscus sabdariffa L. } \\
\text { Malvaceae } \\
\text { Rosella, Mei gui qie }\end{array}$ & Fragmented calyxes [P235] ${ }^{* *}$ & $\begin{array}{l}\text { Hypocholesterolemic, hypolipidemic, anti- } \\
\text { atherosclerotic, anti-lipogenic (Chen et al. } \\
\text { 2003a; Yang et al. 2010; Ibrahim et al. 2013; } \\
\text { García Mesa 2014). }\end{array}$ \\
\hline
\end{tabular}




\begin{tabular}{|c|c|c|}
\hline $\begin{array}{l}\text { Species, families, } \\
\text { local names }\end{array}$ & Products types [Samples] & Biological activity and effects studied \\
\hline $\begin{array}{l}\text { Hypericum perforatum } \mathrm{L} . \\
\text { Hypericaceae } \\
\text { Hypericón }\end{array}$ & $\begin{array}{l}\text { Fragmented aerial parts } \\
{[\mathrm{H} 010][\mathrm{H} 119]}\end{array}$ & $\begin{array}{l}\text { Hypocholesterolemic, hypolipidemic, anti- } \\
\text { dyslipidemic, anti-atherogenic (Zou et al. } \\
\text { 2005; Ineedi and Kumar 2009; Husain et al. } \\
\text { 2011; Asgary et al. 2012). }\end{array}$ \\
\hline $\begin{array}{l}\text { Jodina rhombifolia (Hook. \& } \\
\text { Arn.) Reissek } \\
\text { Santalaceae } \\
\text { Sombra de toro }\end{array}$ & Fragmented leaves [H149] & No data. \\
\hline $\begin{array}{l}\text { Lepidium meyenii Walp. } \\
\text { Brassicaceae } \\
\text { Maca }\end{array}$ & $\begin{array}{l}\text { Fragmented underground } \\
\text { organs [H008] } \\
\text { Powdered underground } \\
\text { organs [H160]* [P271]* [H036] } \\
\text { Tincture [H297] } \\
\text { Capsules [P278]* }[\mathrm{H} 178]\end{array}$ & $\begin{array}{l}\text { Hypocholesterolemic, hypolipidemic, } \\
\text { anti-atherogenic (Vecera et al. 2007; Oré } \\
\text { Sifuentes 2008; Puentes and Hurrell 2015). }\end{array}$ \\
\hline $\begin{array}{l}\text { Linum usitatissimum } \mathrm{L} . \\
\text { Linaceae } \\
\text { Lino }\end{array}$ & $\begin{array}{l}\text { Seeds [H061] [H104] } \\
\text { Powdered seeds [H106] [H316] }\end{array}$ & $\begin{array}{l}\text { Hypocholesterolemic, hypolipidemic, } \\
\text { anti-atherosclerotic (Cunnane et al. 1993; } \\
\text { Muir et al. 1999; Tomaz Pacheco et al. 2011; } \\
\text { Saravanan and Ignacimuthu 2015). }\end{array}$ \\
\hline $\begin{array}{l}\text { Lupinus albus L. } \\
\text { Leguminosae } \\
\text { Lupino }\end{array}$ & Tablets [H308] & $\begin{array}{l}\text { Hypocholesterolemic, hypolipidemic, anti- } \\
\text { dyslipidemic, anti-atherosclerotic (Viveros } \\
\text { et al. 2007; Marchesi et al. 2008; Sewani- } \\
\text { Rusike et al. 2015). }\end{array}$ \\
\hline $\begin{array}{l}\text { Lycium barbarum L. } \\
\text { Solanaceae } \\
\text { Goji }\end{array}$ & Fruits $[\mathrm{D} 001]^{* *}[\mathrm{H} 037]$ & $\begin{array}{l}\text { Hypocholesterolemic, hypolipidemic, anti- } \\
\text { atherosclerotic (Luo et al. 2004; Bidkar et al. } \\
\text { 2011; Jiang 2011; Hurrell et al. 2013; Shafi } \\
\text { and Tabassum 2013). }\end{array}$ \\
\hline $\begin{array}{l}\text { Mangifera indica L. } \\
\text { Anacardiaceae } \\
\text { Mango }\end{array}$ & Fragmented leaves [H427] & $\begin{array}{l}\text { Hypocholesterolemic, hypolipidemic, anti- } \\
\text { atherogenic (Muruganandan et al. 2005; } \\
\text { Hossain et al. 2010). }\end{array}$ \\
\hline $\begin{array}{l}\text { Marrubium vulgare } \mathrm{L} . \\
\text { Lamiaceae } \\
\text { Marrubio }\end{array}$ & $\begin{array}{l}\text { Fragmented aerial parts } \\
\text { [C094] }\end{array}$ & $\begin{array}{l}\text { Hypocholesterolemic, hypolipidemic,anti- } \\
\text { dyslipidemic (Roghani et al. 2005; Shafi and } \\
\text { Tabassum 2013; Elberry et al. 2015). }\end{array}$ \\
\hline $\begin{array}{l}\text { Matricaria chamomilla L. [= } \\
\text { M. recutita L.] } \\
\text { Asteraceae } \\
\text { Manzanilla }\end{array}$ & $\begin{array}{l}\text { Fresh plants [B427]* } \\
\text { Fragmented aerial parts } \\
\text { [H089] } \\
\text { Herbal tea in bags [H016] } \\
\text { Tincture [H357] }\end{array}$ & $\begin{array}{l}\text { Hypocholesterolemic, hypolipidemic, anti- } \\
\text { dyslipidemic (McKay and Blumberg 2006; } \\
\text { Al-Bayati 2012; Al-Musa and Al-Hashem } \\
\text { 2014; Rafraf et al. 2015). }\end{array}$ \\
\hline $\begin{array}{l}\text { Medicago sativa L. } \\
\text { Leguminosae } \\
\text { Alfalfa }\end{array}$ & $\begin{array}{l}\text { Fragmented aerial parts } \\
\text { [H086] } \\
\text { Herbal tea in bags (mixture) } \\
\text { [H046] [H411] } \\
\text { Tablets [H309] } \\
\text { Liquid extract [H310] }\end{array}$ & $\begin{array}{l}\text { Hypocholesterolemic, hypolipidemic, anti- } \\
\text { atherosclerotic (Khaleel et al. 2005; Bidkar et } \\
\text { al. 2011; Shi et al. 2014; Zhou et al. 2014). }\end{array}$ \\
\hline $\begin{array}{l}\text { Melissa officinalis L. } \\
\text { Lamiaceae } \\
\text { Melisa, toronjil }\end{array}$ & $\begin{array}{l}\text { Fragmented aerial parts } \\
\text { [C014] } \\
\text { Tincture [H320] }\end{array}$ & $\begin{array}{l}\text { Hypocholesterolemic, hypolipidemic, anti- } \\
\text { dyslipidemic (Karimi et al. 2010; Weidner et } \\
\text { al. 2014; Zarei et al. 2015). }\end{array}$ \\
\hline $\begin{array}{l}\text { Mentha } \times \text { piperita L. } \\
\text { Lamiaceae } \\
\text { Menta piperita, menta }\end{array}$ & $\begin{array}{l}\text { Fragmented aerial parts } \\
{[\mathrm{C} 124]} \\
\text { Herbal tea in bags [H028] } \\
\text { Herbal teain bags (mixture) } \\
\text { [H065] }\end{array}$ & $\begin{array}{l}\text { Hypocholesterolemic, hypolipidemic (Mani } \\
\text { Badal et al. 2011; Johari et al. 2015). }\end{array}$ \\
\hline
\end{tabular}




\begin{tabular}{|c|c|c|}
\hline $\begin{array}{l}\text { Species, families, } \\
\text { local names }\end{array}$ & Products types [Samples] & Biological activity and effects studied \\
\hline $\begin{array}{l}\text { Morinda citrifolia L. } \\
\text { Rubiaceae } \\
\text { Noni }\end{array}$ & $\begin{array}{l}\text { Fresh fruits [P218]* } \\
\text { Powdered fruits [H161]* } \\
\text { [RF80] } \\
\text { Capsules [H162]* [H379] } \\
\text { Liquid extract [PN03] }\end{array}$ & $\begin{array}{l}\text { Hypocholesterolemic, hypolipidemic, anti- } \\
\text { dyslipidemic, anti-atherosclerotic (Kamiya et } \\
\text { al. 2004; Mandukhail et al. 2010; Nayak et al. } \\
\text { 2011; Lin et al. 2012; Lee et al. 2012). }\end{array}$ \\
\hline $\begin{array}{l}\text { Moringa oleifera Lam. } \\
\text { Moringaceae } \\
\text { Moringa }\end{array}$ & Fragmented leaves [H432] & $\begin{array}{l}\text { Hypocholesterolemic, hypolipidemic, anti- } \\
\text { dyslipidemic, anti-atherosclerotic (Ghasi et } \\
\text { al. 2000; Chumark et al. 2008; Mbikay 2012; } \\
\text { Dhaliya et al. 2013). }\end{array}$ \\
\hline $\begin{array}{l}\text { Myrciaria dubia (Kunth) } \\
\text { McVaugh } \\
\text { Myrtaceae } \\
\text { Camu-camu }\end{array}$ & Capsules [H602] & $\begin{array}{l}\text { Hypocholesterolemic, hypolipidemic } \\
\text { (Nascimento et al. 2013; Langley et al. 2015). }\end{array}$ \\
\hline $\begin{array}{l}\text { Olea europaea } \mathrm{L} . \\
\text { Oleaceae } \\
\text { Olivo }\end{array}$ & Leaves [H191] & $\begin{array}{l}\text { Hypocholesterolemic, hypolipidemic, anti- } \\
\text { atherosclerotic (Miranda Velásquez 2010; } \\
\text { Susalit et al. 2011). }\end{array}$ \\
\hline $\begin{array}{l}\text { Panax ginseng C. A. Mey. } \\
\text { Araliaceae } \\
\text { Ginseng }\end{array}$ & $\begin{array}{l}\text { Fragmented roots [H114] } \\
\text { Herbal tea in bags (mixture) } \\
\text { [H046] } \\
\text { Tablets [H319] } \\
\text { Capsules (mixture) [H323] }\end{array}$ & $\begin{array}{l}\text { Hypocholesterolemic, hypolipidemic, anti- } \\
\text { atherosclerotic (Yamamoto et al. 1983; Lee } \\
\text { et al. 2013; Kawase et al. 2014). }\end{array}$ \\
\hline $\begin{array}{l}\text { Paullinia cupana Kunth } \\
\text { Sapindaceae } \\
\text { Guaraná }\end{array}$ & $\begin{array}{l}\text { Seeds }[\mathrm{H} 258] \\
\text { Tablets [H311] }\end{array}$ & $\begin{array}{l}\text { Hypocholesterolemic, hypolipidemic, anti- } \\
\text { dyslipidemic,anti-atherosclerotic (Pizziolo et } \\
\text { al. 2011; Lima Portella et al. 2013). }\end{array}$ \\
\hline $\begin{array}{l}\text { Peumus boldus Molina } \\
\text { Monimiaceae } \\
\text { Boldo }\end{array}$ & $\begin{array}{l}\text { Fragmented leaves [C010] } \\
\text { Herbal tea in bags [H017] } \\
\text { Herbal tea in bags (mixture) } \\
\text { [H047] [H412] } \\
\text { Tablets (mixture) [H501] }\end{array}$ & $\begin{array}{l}\text { Hypocholesterolemic, anti-atherosclerotic } \\
\text { (Santanam et al. 2004; Falé et al. 2014). }\end{array}$ \\
\hline $\begin{array}{l}\text { Phyllanthus niruri L. } \\
\text { Euphorbiaceae } \\
\text { Chancapiedra }\end{array}$ & $\begin{array}{l}\text { Fragmented aerial parts } \\
{[\mathrm{P} 195]^{*}[\mathrm{P} 205]} \\
\text { Capsules }[\mathrm{H} 441]^{*}[\mathrm{H} 447]\end{array}$ & $\begin{array}{l}\text { Hypocholesterolemic, hypolipidemic } \\
\text { (Khanna et al. 2002; Barboza et al. 2009; } \\
\text { Megraj et al. 2011). }\end{array}$ \\
\hline $\begin{array}{l}\text { Plantago major L. } \\
\text { Plantaginaceae } \\
\text { Llantén }\end{array}$ & Fragmented leaves [H208] & $\begin{array}{l}\text { Hypocholesterolemic, anti-atherogenic } \\
\text { (Angarskaya and Sokolova 1963; García- } \\
\text { Lazo et al. 2015). }\end{array}$ \\
\hline $\begin{array}{l}\text { Plantago ovata Forssk. } \\
\text { Plantaginaceae } \\
\text { Psyllium }\end{array}$ & $\begin{array}{l}\text { Seeds }[\mathrm{H} 393]^{* *} \\
\text { Powdered seeds }[\mathrm{H} 325]\end{array}$ & $\begin{array}{l}\text { Hypocholesterolemic, hypolipidemic, anti- } \\
\text { dyslipidemic (Wärnberg et al. 2009; Solàet } \\
\text { al. 2007, 2010). }\end{array}$ \\
\hline $\begin{array}{l}\text { Plukenetia volubilis L. } \\
\text { Euphorbiaceae } \\
\text { Sacha inchi }\end{array}$ & $\begin{array}{l}\text { Seeds [D005] } \\
\text { Powdered seeds [P249]* } \\
\text { Capsules (extract) [D003] } \\
\text { Capsules (oil) [H301] }\end{array}$ & $\begin{array}{l}\text { Hypocholesterolemic, hypolipidemic, } \\
\text { anti-dyslipidemic, anti-atherosclerotic } \\
\text { (Garmendia et al. 2011; Hurrell et al. 2013; } \\
\text { Puentes and Hurrell 2015). }\end{array}$ \\
\hline $\begin{array}{l}\text { Polygonum aviculare } \mathrm{L} . \\
\text { Polygonaceae } \\
\text { Sanguinaria }\end{array}$ & $\begin{array}{l}\text { Fragmented aerial parts } \\
{[\mathrm{H} 197]}\end{array}$ & $\begin{array}{l}\text { Hypocholesterolemic, hypolipidemic, anti- } \\
\text { lipogenic, anti-atherosclerotic (Sung et al. } \\
\text { 2013; Park et al. 2014). }\end{array}$ \\
\hline $\begin{array}{l}\text { Psidium guajava L. } \\
\text { Myrtaceae } \\
\text { Arazá }\end{array}$ & Fragmented leaves [H260] & $\begin{array}{l}\text { Hypocholesterolemic, hypolipidemic } \\
\text { (Deguchi and Miyazaki 2010; Dhaliya et al. } \\
\text { 2013; Puentes and Hurrell 2015). }\end{array}$ \\
\hline $\begin{array}{l}\text { Rehmannia glutinosa } \\
\text { (Gaertn.) DC. } \\
\text { Orobanchaceae } \\
\text { Gan di huang }\end{array}$ & Fragmented roots $[\mathrm{H} 448]^{* *}$ & $\begin{array}{l}\text { Hypocholesterolemic, hypolipidemic (Poon } \\
\text { et al. 2011; Zhou et al. 2015). }\end{array}$ \\
\hline
\end{tabular}




\begin{tabular}{|c|c|c|}
\hline $\begin{array}{l}\text { Species, families, } \\
\text { local names }\end{array}$ & Products types [Samples] & Biological activity and effects studied \\
\hline $\begin{array}{l}\text { Rheum officinale Baill. } \\
\text { Polygonaceae } \\
\text { Ruibarbo }\end{array}$ & $\begin{array}{l}\text { Fragmented rhizomes [H216] } \\
\text { [H435] }\end{array}$ & $\begin{array}{l}\text { Hypocholesterolemic, hypolipidemic, anti- } \\
\text { dyslipidemic, anti-atherosclerotic (Nada et } \\
\text { al. 1997; Gao et al. 2010; Zhong et al. 2012). }\end{array}$ \\
\hline $\begin{array}{l}\text { Salvia hispanica L. } \\
\text { Lamiaceae } \\
\text { Chía }\end{array}$ & $\begin{array}{l}\text { Seeds [H042] [H113] } \\
\text { Powdered seeds [H107] [H313] }\end{array}$ & $\begin{array}{l}\text { Hypocholesterolemic, hypolipidemic, anti- } \\
\text { atherosclerotic (Vázquez-Ovando et al. 2009; } \\
\text { Ali et al. 2012; Sierra et al. 2015). }\end{array}$ \\
\hline $\begin{array}{l}\text { Salvia officinalis L. } \\
\text { Lamiaceae } \\
\text { Salvia }\end{array}$ & Fragmented leaves [C015] & $\begin{array}{l}\text { Hypocholesterolemic, hypolipidemic } \\
\text { (Ibrahim et al. 2013; García Mesa 2014). }\end{array}$ \\
\hline $\begin{array}{l}\text { Satureja hortensis } \mathrm{L} . \\
\text { Lamiaceae } \\
\text { Ajedrea }\end{array}$ & $\begin{array}{l}\text { Fragmented leaves [C028] } \\
{[\mathrm{H} 070]}\end{array}$ & $\begin{array}{l}\text { Hypocholesterolemic, hypolipidemic } \\
\text { (Mchedlishvili et al. 2005; Momtaz and } \\
\text { Abdollahi 2008). }\end{array}$ \\
\hline $\begin{array}{l}\text { Schisandra chinensis } \\
\text { (Turcz.) Baill. } \\
\text { Schisandraceae } \\
\text { Eschisandra }\end{array}$ & $\begin{array}{l}\text { Fruits }[\mathrm{RF} 59]^{* *}[\mathrm{P} 208] \\
\text { Capsules (mixture) }[\mathrm{H} 323]\end{array}$ & $\begin{array}{l}\text { Hypocholesterolemic (Panossian and } \\
\text { Wikman 2008; Pan et al. 2012). }\end{array}$ \\
\hline $\begin{array}{l}\text { Silybum marianum (L.) } \\
\text { Gaertn. } \\
\text { Asteraceae } \\
\text { Cardo mariano }\end{array}$ & $\begin{array}{l}\text { Seeds [H154] } \\
\text { Capsules [H354] }\end{array}$ & $\begin{array}{l}\text { Hypocholesterolemic, hypolipidemic, anti- } \\
\text { dyslipidemic, anti-atherosclerotic (Krecman } \\
\text { et al. 1998; Huseini et al. 2006; Radjabian } \\
\text { et al. 2010; Derosa et al. 2013; Hurrell and } \\
\text { Puentes 2013). }\end{array}$ \\
\hline $\begin{array}{l}\text { Smallanthus sonchifolius } \\
\text { (Poepp. \& Endl.) H. Rob. } \\
\text { Asteraceae } \\
\text { Yacón }\end{array}$ & $\begin{array}{l}\text { Fresh roots [H6891]* } \\
\text { Fragmented leaves [H332] } \\
\text { Tincture [H285] } \\
\text { Capsules [H286] [H293] } \\
\text { Liquid extract [P275] }\end{array}$ & $\begin{array}{l}\text { Hypocholesterolemic, hypolipidemic (Miura } \\
\text { et al. 2004; Valencia 2005; Habib et al. 2011; } \\
\text { Hurrell et al. 2013; Puentes and Hurrell } \\
\text { 2015). }\end{array}$ \\
\hline $\begin{array}{l}\text { Stevia rebaudiana (Bertoni) } \\
\text { Bertoni } \\
\text { Asteraceae } \\
\text { Yerba dulce }\end{array}$ & $\begin{array}{l}\text { Fresh plants [B415]* } \\
\text { Fragmented leaves [H198] } \\
\text { Tincture [H350] } \\
\text { Powdered extract [H116] } \\
\text { Liquid extract [H317] }\end{array}$ & $\begin{array}{l}\text { Hypocholesterolemic, hypolipidemic, anti- } \\
\text { dyslipidemic, anti-atherosclerotic (Savita et } \\
\text { al. 2004; Geeraert et al. 2010; Hossain et al. } \\
\text { 2011; Singh and Garg 2014). }\end{array}$ \\
\hline $\begin{array}{l}\text { Taraxacum officinale Weber } \\
\text { ex F.H. Wigg. } \\
\text { Asteraceae } \\
\text { Diente de león, amargón }\end{array}$ & $\begin{array}{l}\text { Fragmented aerial parts } \\
{[\mathrm{H} 100]^{*}[\mathrm{C} 087]} \\
\text { Tincture }[\mathrm{H} 337]\end{array}$ & $\begin{array}{l}\text { Hypocholesterolemic, hypolipidemic, anti- } \\
\text { atherosclerotic (Choi et al. 2010; Alabi et al. } \\
\text { 2013; Hurrell and Puentes 2013). }\end{array}$ \\
\hline $\begin{array}{l}\text { Tessaria integrifolia Ruiz \& } \\
\text { Pav. } \\
\text { Asteraceae } \\
\text { Pájaro bobo }\end{array}$ & $\begin{array}{l}\text { Fragmented aerial parts } \\
{[\mathrm{H} 227][\mathrm{H} 335]} \\
\text { Tincture }[\mathrm{H} 340]\end{array}$ & No data. \\
\hline $\begin{array}{l}\text { Trigonella foenum-graecum } \\
\text { L. } \\
\text { Leguminosae } \\
\text { Fenogreco }\end{array}$ & $\begin{array}{l}\text { Seeds [H452] } \\
\text { Powdered seeds [H062] }\end{array}$ & $\begin{array}{l}\text { Hypocholesterolemic, hypolipidemic, anti- } \\
\text { dyslipidemic, anti-atherogenic (Nada et } \\
\text { al. 1997; Ibrahim et al. 2013; Kumar and } \\
\text { Bhandari 2013; Yadav and Baquer 2014). }\end{array}$ \\
\hline $\begin{array}{l}\text { Trixis divaricata } \\
\text { (Kunth) Spreng. [=T. } \\
\text { antimenorrhoea } \\
\text { (Schrank) Mart. ex Kuntze] } \\
\text { Asteraceae } \\
\text { Contrayerba }\end{array}$ & $\begin{array}{l}\text { Fragmented aerial parts } \\
{[\mathrm{P} 146]}\end{array}$ & Hypocholesterolemic (Granato et al. 2013). \\
\hline $\begin{array}{l}\text { Uncaria tomentosa (Willd.) } \\
\text { DC. } \\
\text { Rubiaceae } \\
\text { Uña de gato }\end{array}$ & $\begin{array}{l}\text { Bark (chunks) [H109] } \\
\text { Bark (splinters) [H110] } \\
\text { Tincture [H273] } \\
\text { Capsules [H378] }\end{array}$ & $\begin{array}{l}\text { Hypocholesterolemic, anti-atherosclerotic } \\
\text { (Potawale et al. 2008; Pizziolo et al. 2011). }\end{array}$ \\
\hline
\end{tabular}




\begin{tabular}{|c|c|c|}
\hline $\begin{array}{l}\text { Species, families, } \\
\text { local names }\end{array}$ & Products types [Samples] & Biological activity and effects studied \\
\hline $\begin{array}{l}\text { Vaccinium corymbosum L. } \\
\text { Aiton } \\
\text { Ericaceae } \\
\text { Arándano, arándano azul }\end{array}$ & $\begin{array}{l}\text { Fragmented leaves [H029] } \\
\text { Tablets [H407] }\end{array}$ & $\begin{array}{l}\text { Hypocholesterolemic, hypolipidemic, anti- } \\
\text { atherogenic (Kim et al. 2010; Çoban et al. } \\
\text { 2013; Liang et al. 2013). }\end{array}$ \\
\hline $\begin{array}{l}\text { Vaccinium macrocarpon } \\
\text { Aiton } \\
\text { Ericaceae } \\
\text { Arándano rojo }\end{array}$ & Tablets [H406] & $\begin{array}{l}\text { Hypocholesterolemic, hypolipidemic, anti- } \\
\text { atherosclerotic (Shabrova et al. 2011; Anhê } \\
\text { et al. 2015). }\end{array}$ \\
\hline $\begin{array}{l}\text { Xanthium spinosum } \mathrm{L} . \\
\text { Asteraceae } \\
\text { Cepa caballo, abrojo }\end{array}$ & $\begin{array}{l}\text { Fragmented aerial parts } \\
\text { [H127] } \\
\text { Tincture }[\mathrm{H} 344]\end{array}$ & No data. \\
\hline $\begin{array}{l}\text { Zingiber officinale Roscoe } \\
\text { Zingiberaceae } \\
\text { Jengibre }\end{array}$ & $\begin{array}{l}\text { Fragmented rhizomes [H453] } \\
\text { Powdered rhizomes [H454] } \\
\text { Capsules (mixture) [P170] }\end{array}$ & $\begin{array}{l}\text { Hypocholesterolemic, hypolipidemic, anti- } \\
\text { dyslipidemic, anti-atherosclerotic (Verma et } \\
\text { al. 2004; Bhandari et al. 2005; Megraj et al. } \\
\text { 2011; Bidkar et al. 2011; Ibrahim et al. 2013; } \\
\text { García Mesa 2014). }\end{array}$ \\
\hline
\end{tabular}

Ofthe 82 speciessurveyed,23(28\%) are aromatic species consumed both as therapeutics and food condiment and/or beverages flavoring: Achillea millefolium, Achyrocline satureioides, Acorus calamus, Allium sativum, Aloysia citriodora, Alpinia officinarum, Angelica archangelica, Artemisia absinthium, Baccharis articulata, B. trimera, Bixa Orellana, Curcuma longa, Cymbopogon citratus, Foeniculum vulgare, Marrubium vulgare, Matricaria chamomilla, Melissa officinalis, Mentha x piperita, Peumus boldus, Salvia officinalis, Satureja hortensis, Trigonella foenum-graecum, and Zingiber officinale.

Only 7 species (8.5\%) have no academic studies that validate their hypocholesterolemic effect. These species are: Aloysia citriodora, Aspidosperma quebracho-blanco, Baccharis articulata, Heimia salicifolia, Jodina rhombifolia, Tessaria integrifolia, and Xanthium spinosum. It would be desirable to encourage future validation studies for these species.

Products of 10 species (12.2\% of total) are related to the Chinese immigrants segment. Half of these species are commercialized only in the Barrio Chino: Angelica sinensis, Atractylodes lancea, Eucommia ulmoides, Hibiscus sabdariffa, and Rehmannia glutinosa. The remaining five species have products sold in the restricted circuit of the Barrio Chino, but also have products that are marketed in the general commercial circuit: Astragalus mongholicus, Garcinia gummi-gutta,
Lycium barbarum, Plantago ovata, and Schisandra chinensis.

Products belonging to 17 species $(20.8 \%$ of total) are sold in the Bolivian market of Liniers: Achyrocline satureioides, Allium ampeloprasum, Artemisia absinthium, Baccharis articulata, B. trimera, Bixa orellana, Centella asiatica, Cymbopogon citratus, Cynara cardunculus, Lepidium meyenii, Matricaria chamomilla, Morinda citrifolia, Phyllanthus niruri, Plukenetia volubilis, Smallanthus sonchifolius, Stevia rebaudiana, and Taraxacum officinale. All these species are also represented through different products in the general commercial circuit.

Of the total of 82 species surveyed, 55 species $(67 \%)$ have products that are marketed only in the general commercial circuit.

\section{DISCUSSION AND CONCLUSIONS}

The theoretical and methodological basis of the research line assumes that: 1) the local BK orients the strategies of selection and use of plant products; 2) these strategies are evident in the circulation of those products within local commercial circuits; 3) since there is no direct access to the $\mathrm{BK}$, its reconstruction is possible starting from the assessment of the circulation of those plant products. As noted above, plant products circulating only inside the restricted 
commercial circuit of the immigrant segments are considered invisible to most of the urban population. On the other hand, plant products circulating inside the general commercial circuit are considered visible to all urban inhabitants. In this context, invisible plant products are related to the BK linked to traditions, and visible plant products are related to the non traditional BK. The dynamics of urban BK is expressed through the passage of plant products from the restricted circuit of immigrants towards general commercial circuit, so those products acquire visibility. That 'passage' of plant products involves a contextual change that we call visualization process. This process implies the transmission of BK that is expressed in the diffusion of the products, mainly performed by the mass media and specialized stores as the dietéticas. The evaluation of the results of this contribution on the medicinal plants used as hypocholesterolemic allows us to illustrate the dynamics of the local BK.

With regard to the Chinese immigrants segment, plant products of five species are exclusive of this restricted commercial circuit, therefore, these species are invisible. Other five species have products sold in the restricted circuit of the Barrio Chino, but also have products that are marketed in the general commercial circuit. In these cases, particular products remain invisible, but the species are visible because other plant products are marketed in the general commercial circuit. Dried roots of Astragalus mongholicus, dried fruits of Garcinia gummi-gutta, and seeds of Plantago ovata are exclusive (invisible) products of the Chinese segment. Fragmented roots and capsules of $A$. mongholicus, tincture, tablets and capsules of G. gummi-gutta, and powdered seeds of $P$. ovata are products marketed in the general commercial circuit (visible). Dried fruits of Lycium barbarum and Schisandra chinensis are present both in general and restricted circuits. Should be noted that products represented in the general circuit are mostly processed, and therefore more adequate for the urban lifestyle, because are easier to consume. Through the products, the species acquire visibility, and enter the context of the non-traditional BK. The products diffusion can be a quick process, enhanced by the media, as was previously indicated for L. barbarum (Hurrell et al. 2013). Moreover, the widest variety of products of G. gummi-gutta is evidence of a wider diffusion and, at the same time, a major permanence in the general commercial circuit.

For the segment of Bolivian immigrants, the 17 species marketed in the Bolivian market of Liniers are also sold in the general commercial circuit, but through different products. In this sense, it is important to highlight that although the species are visible, their particular products can be visible or invisible. For instance, from the standpoint of particular products, fresh plants of Artemisia absinthium, Baccharis articulata, B. trimera, Matricaria chamomilla, and Stevia rebaudiana, fresh fruits of Morinda citrifolia, fresh roots of Smallanthus sonchifolius, powdered seeds of Plukenetia volubilis, and fragmented aerial parts of Centella asiatica, are exclusive of the Bolivian market, thus, are invisible. As in the case of the Chinese immigrants segment, products disseminated in the general circuit that were introduced from the segment Bolivian immigrants mostly correspond to tinctures, tablets, capsules, extracts, ie, products easy to consume, preferred by urban inhabitants. The Bolivian market of Liniers constitutes a source of diverse plant products that enrich local urban diversity, both products as their related BK (Pochettino et al. 2012). In the last decade, the research line of the LEBA has studied the entry of several plant products into the Bolivian market of Liniers coming from Peru and Bolivia. Thereafter, these products have entered into the general commercial circuit of the study area, so the species became visible. Such is the case of Lepidium meyenii and M. citrifolia (Arenas et al. 2011), P. volubilis and S. sonchifolius (Hurrell et al. 2013), and Allium ampeloprasum (Puentes and Hurrell 2015). Except $A$. ampeloprasum, with only one product recently disseminated and at present under expansion, the other species have diverse products, and their diffusion is wider and their permanence time is longer in local general commercial circuit.

Regarding to general commercial circuit, 55 species used as hypocholesterolemic are only presented in this context related to the nontraditional urban BK. Thus, are visible with greater 
or lesser level of diffusion. Some of these species, like Aloysia citriodora, Aspidosperma quebrachoblanco, Handroanthus impetiginosus, Jodina rhombifolia, and Plantago major, have their roots in the popular medicine of Argentina, which favors its diffusion in the commercial circuit. Other species such as Ginkgo biloba, Glycyrrhiza glabra, Panax ginseng and Uncaria tomentosa have wide circulation as dietary supplements. They are products easy to consume, fact that increases their chances of diffusion. Others species have recently been introduced into the local urban scenario, like Euterpe oleracea, whose products have more poor dissemination (Hurrell et al. 2013).

In summary, of the cholesterol-lowering 82 treated species, 77 (93.9\%) are visible, including 55 species marketed only in the general commercial circuit, 17 species of the Bolivian market and 5 species of the Barrio Chino that are also represented in that circuit. Only 5 species (6.1\%) of the Chinese immigrants segment are invisible. It is possible that these species acquire visibility in the future, if we assume that could enter the general commercial circuit, as happened recently with Lycium barbarum (Hurrell et al. 2013). In this framework, the restricted circuits of immigrants segments act as sources of species and their associated knowledge that increase the local biocultural diversity.

The interest of the urban population by the problematic of cholesterol, linked to the risk of cardiovascular disorders and diseases as diabetes and obesity is reflected in the high visibility of different species used as cholesterol-lowering and the variety of products available. A similar situation occurs with other categories of medicinal plants related to the lifestyle of large cities: slimming, antioxidants, adaptogens, cognitive enhancers, and aphrodisiacs (Arenas et al. 2011, 2015; Hurrell et al. 2013, 2015; Hurrell \& Puentes, 2013; Hurrell 2014). In this context, this paper constitutes a new contribution in order to understand the complexity of the botanical knowledge dynamics for the study area.

Moreover, this paper also constitutes a contribution to the field of the ethnobotany of the multicultural urban contexts regarding with its theoretical-methodological framework. Because the botanical knowledge (both non-traditional and linked to traditions) guides the strategies of selection and utilization of species and products, the analysis of circulating products constitute an adequate methodological tool to assess the characteristics of the local botanical knowledge and its transmission inside the multicultural urban contexts, in which the mass media are actively involved.

\section{ACKNOWLEDGMENTS}

The authors thank Dr. María Lelia Pochettino and the integrants of LEBA, as well as the informants that were part of the field works. This research line is carried out with financial support of the Universidad Nacional de La Plata and the Consejo Nacional de Investigaciones Cientificas y Técnicas (CONICET), Argentina.

\section{REFERENCES}

1. Afonso MS, Silva AM, Carvalho EBT, RivelliDP, Barros SBM, Rogero MM, Lottenberg AM, Torres RP, Mancini-Filho J (2013). Phenolic compounds from Rosemary (Rosmarinus officinalis $\mathrm{L}$.) attenuate oxidative stress and reduce blood cholesterol concentrations in diet-induced hypercholesterolemic rats. Nutrition \& Metabolism 10:19, http://www.nutritionandmetabolism.com/content/10/1/19.

2. Al-Bayati AJ (2012) Study the effect of chamomile on hyperlipidaemias in Guinea pigs. Kufa J Vet Med Sci 3(2):61-65.

3. Al-Musa H, Al-Hashem F (2014) Hypoglycemic, hepato-renal and antioxidant potential effects of chamomile recutita flowers ethanolic extract in streptozotocin-diabetic rats. Amer $\mathrm{J}$ Pharmacol Toxicol 9(1):1-12.

4. Alabi M, Sunday R, Olowokere T, Kareem FA, Osanaiye F (2013) Effect of bitters on the body weight, lipid profile, catalase, and lipid peroxidation in experimental animals.J Med Sci13:62-66.

5. Albuquerque UP, Cruz da Cunha LVF, Lucena RFP, Alves RRN (eds.) (2014) Methods and techniques in Ethnobiology and Ethnoecology. Springer-Humana Press, New York.

6. Alegría Ezquerra E, Castellano Vázquez JM, Alegría Barrero A (2008) Obesidad, síndrome metabólico y diabetes: implicaciones cardiovasculares y actuación terapéutica. Rev Esp Cardiol 61(7):752-764

7. Ali NM, Yeap SK, Ho WY, Beh BK, Tan SW and Tan SG (2012) The promising future of chia, Salvia hispanica L. J Biomed Biotechnol 2012:171956, doi: 10.1155/2012/171956.

8. Andallu B, Ramya V (2007) Antihyperglycemic, cholesterollowering and HDL-raising effects of Cumin (Cuminum cyminum) seeds in type - 2 diabetes. J Nat Remedies 7(1):142-149.

9. Angarskaya MA, Sokolova VE (1963) The effect of Plantain (Plantago major) on the course of experimental atherosclerosis in rabbits. Bull Experim Biol Med 53(4):410412. 
10. Anhê $F$, Roy D, Pilon G, Dudonné $S$, Matamoros $S$, Varin $T$, Garofalo C, Moine Q, Desjardins Y, Levy E, Marette A (2015) A polyphenol-rich cranberry extract protects from diet-induced obesity, insulin resistance and intestinal inflammation in association with increased Akkermansia sp. population in the gut microbiota of mice. Gut $64(6): 872-883$.

11. Arenas PM, Cristina I, Puentes JP, Buet Costantino F, Hurrell JA, Pochettino, ML (2011) Adaptógenos: plantas medicinales tradicionales comercializadas como suplementos dietéticos en la conurbación Buenos Aires-La Plata (Argentina). Bonplandia (Corrientes) 20(2):251-264.

12. Arenas PM, Doumecq B, Puentes JP, Hurrell JA (2015) Algas y plantas comercializadas como adelgazantes en el Área Metropolitana de Buenos Aires, Argentina. Gaia Scientia (in press).

13. Asgary S, Jafari Dinani N, Madani H, Mahzoni P, Naderi G (2007) Effect of Glycyrrhiza glabra extract on aorta wall atherosclerotic lesion in hypercholesterolemic rabbits. Pak J Nutr 6(4):313-317.

14. Asgary S, Solhpour A, Parkhideh S, Madani H, Mazouni $P$, Kabiri N (2012) Effect of hydroalcoholic extract ofHypericum perforatumon selected traditional and novel biochemical factors of cardiovascular diseases and atherosclerotic lesions in hypercholesterolemic rabbits. A comparison between the extract and lovastatin. J Pharm Bioallied Sci 4(3):212-218.

15. Ateş A, Esen Gürsel F, Bilal T, Altıner A (2012) Effect of dietary Garcinia cambogia extract on serum lipid profile and serum enzymes in rats fed high-lipid diet. Iranian J Vet Res 13(1):1-7.

16. Badgujar SB, Patel VV, Bandivdekar AH (2014) Foeniculum vulgare Mill.ARreview of its botany, phytochemistry, pharmacology,contemporary application, and toxicology. BioMedRes Int 2014: 842674, doi:http://dx.doi. org/10.1155/2014/842674.

17. Barboza G, Cantero J, Nuñez CO, Pacciaroni A, Ariza Espinar L (2009) Medicinal plants: A general review and a phytochemical and ethnopharmacological screening of the native Argentine Flora. Kurtziana 34(1-2):7-365.

18. Bhandari U, Kanojia R, Pillai KK (2005) Effect of ethanolic extract of Zingiber officinale on dyslipidaemia in diabetic rats. J Ethnopharmacol 97(2):227-230.

19. Bidkar JS, Ghanwat DD, Dama GY, Bidkar SJ, Bhujbal MD, Shende VS (2011) A global review on plants with hypolipidemic activity. Deccan J Pharmacol 2(1):1-19.

20. Blanco-Castro E (1996) Ideas metodológicas relativas al trabajo de campo etnobotánico. Monogr Jard Bot Córdoba (España) 3:89-91.

21. Bongiolo AM (2008) Efeito do extrato hidroalcoólico de Eugenia uniflora L. (Myrtaceae) sobre a hiperglicemia e dislipidemia de ratos diabéticos induzidos por aloxana. Universidade do Extremo Sul Catarinense, Criciúma.

22. Bordia A, Verma SK, Srivastava (1998) Effect of garlic (Allium sativum) on blood lipids, blood sugar, fibrinogen and fibrinolytic activity in patients with coronary artery disease. PLEFA $58(4): 257-263$

23. Brown JE, Rice-Evans CA (1998) Luteolin-rich artichoke extracts protect low density lipoprotein from oxidation in vitro. Free Radic Res 29:247-255.

24. Chauhan PK, Pandey IP, Dhatwalia VK (2010) Evaluation of the anti-diabetic effect of ethanolic and methanolic extracts of Centella asiatica leaves extract on alloxan induced diabetic rats. Adv Biol Res 4(1):27-30.
25. Chen CC, Hsu JD, Wang SF, Chiang HC, Yang MY, Kao ES, Ho YC, Wang CJ (2003a) Hibiscus sabdariffa extract inhibits the development of atherosclerosis in cholesterol-fed rabbits. $\mathrm{J}$ Agric Food Chem 51(18):5472-5477.

26. Chen HL, Sheu WH, Tai TS, Liaw YP, Chen YC (2003b) Konjac supplement alleviate hypercholesterolemia and hyperglycemia in type 2 diabetic subjects. A randomized double-blindtrial. J Am Coll Nutr 22(1):36-42.

27. Choi MS, Jung LJ, Kim HJ, Do GM, Jeon SM, Kim SM, Lee MK (2008) Du-zhong (Eucommiaulmoides Oliver) leaf extract mediates hypolipidemic action in hamsters fed a high-fat diet. Am J Chin Med 36:81, doi: 10.1142/S0192415X08005606.

28. Choi UK, Lee OH, Yim JH, Cho CW, Rhee YK, Lim SI, Kim YC (2010) Hypolipidemic and antioxidant effects of dandelion (Taraxacum officinale) root and leaf on cholesterol-fed rabbits. Int J Mol Sci 11(1):67-78.

29. Choi WH, Um MY, Ahn J, Jung CH, Park MK, Ha TY (2014) Ethanolic extract of Taheebo attenuates increase in body weight and fatty liver in mice fed a high-fat diet. Molecules 19(10):16013-16023.

30. Chumark $P$, Khunawat $P$, Sanvarinda $Y$, Phornchirasilp $S$, Morales NP, Phivthong-Ngam L, Ratanachamnong P, Srisawat $S$, Pongrapeeporn KU (2008) The in vitro and ex vivo antioxidant properties, hypolipidaemic and antiatherosclerotic activities of water extract of Moringa oleifera leaves. J Ethnopharmacol 116(3):439-446.

31. Çoban J, Evran B, Özkan F, Çevik A, Dogru-Abbasoglu S, Uysal $M$ (2013) Effect of blueberry feeding on lipids and oxidative stress in the serum, liver and aorta of Guinea pigs fed on a highcholesterol diet. Biosci Biotechnol Biochem 77(2):389-391.

32. Costa C, Bidinotto LT, Takahira RK, Salvadori DMF, Barbisan LF, Costa $M$ (2011) Cholesterol reduction and lack of genotoxic or toxic effects in mice after repeated 21-day oral intake of lemongrass (Cymbopogon citratus) essential oil. Food Chem Toxicol 49(9):2268-2272.

33. Cunnane SC, Ganguli S, Menard C, Liede AC, Hamadeh MJ, Chen ZY, Wolever TMS, Jenkins DJA (1993) High a-linolenic acid flaxseed (Linum usitatissimum): some nutritional properties in humans. Br J Nutr 69:443-453.

34. Dah-Nouvlessounon D, Adoukonou-Sagbadja H, Diarrassouba N, Sina H, Adjanohoun A, Inoussa M, Akakpo D, Gbenou JD, Kotchoni S0, Dicko MH, Baba-Moussa L (2015) Phytochemicalanalysis and biological activities of Cola nitida bark. Biochem Res Int 2015:493879, doi: http://dx.doi.org/10.1155/2015/493879.

35. Dalli E, Colomer E, Tormos MC, Cosin-Sales J, Milara J, Esteban E, Sáez G (2011) Crataegus laevigata decreases neutrophil elastase and has hypolipidemic effect: a randomized, doubleblind, placebo-controlled trial. Phytomedicine 18(8-9):769-775.

36. Daradka HM, Badawneh M, Al-Jamal JA, Bataineh Y (2014) Hypolipidemic efficacy of Artemisia absinthium extracts in rabbits. World Appl Sci J 31(8):1415-1421.

37. Deguchi Y, Miyazaki K (2010) Anti-hyperglycemic and antihyperlipidemic effects of guava leaf extract. Nutr Metab (London) 7:9, doi: 10.1186/1743-7075-7-9.

38. Deng $R$ (2009) Food and food supplements with hypocholesterolemic effects. Recent Pat Food Nutr Agric 1:1524.

39. Derosa G, Bonaventura A, Bianchi L, Romano D, D'Angelo A, Fogari E, Maffioli P (2013). Berberis aristata/Silybum marianum fixed combination on lipid profile and insulin secretion in dyslipidemic patients. Expert Opin Biol Ther. 13(11):1495-506. 
40. Devalaraja S, Jain S, Yadav H (2011) Exotic fruits as therapeutic complements for diabetes, obesity and metabolic syndrome. Food Res Int 44(7):1856-1865.

41. Dey P, Khaled KL (2013) An extensive review on Allium ampeloprasum, a magical herb. Int J Sci Res 4(7):371-377.

42. Dhaliya SA, Surya AS, Dawn VT, Betty C, Arun K, Sunil C (2013) A review of hyperlipidemia and medicinal plants. IntJ A PS BMS 2(4):219-237.

43. Divya G, Gajalakshmi S, Mythili S, Sathiavelu A (2011) Pharmacological activities of Acoruscalamus. A review. Asian J Biochem Pharm Res 4(1):57-64.

44. Ekpenyong CE, Davies K, Antai E (2014) Cymbopogon citratus Stapf (DC) extract ameliorates atherogenic cardiovascular Rrisk in diabetes-induced dyslipidemia in rats. $\mathrm{Br} \mathrm{J}$ Med Medical Res 4(28):4695-4709.

45. El-Saadany SS, El-Massry RA, Labib SM, Sitohy MZ (1991) The biochemical role and hypocholesterolaemic potential of the legume Cassia fistula in hypercholesterolaemic rats. Molecular Nutr Food Res 35(8):807-815.

46. Elberry AA, Harraz FM, Ghareib $S A$, Gabr SA, Nagy AA, Abdel-Sattar E (2015) Methanolic extract ofMarrubium vulgareameliorates hyperglycemia and dyslipidemia in streptozotocin-induced diabetic rats. Int $\mathrm{J}$ Diabetes Mellitus 3(1):37-44.

47. Espiña $\mathrm{D}$, Carvalho $\mathrm{F}$, Zanini $\mathrm{D}$, Schlemmer J, Coracini J, Rubin $M$, Morsch V, Schetinger MR, Leal D, Baiotto CR, Jaques JA (2012) A more accurate profile of Achyrocline satureioides hypocholesterolemic activity. Cell Biochem Funct 30(4):347-353.

48. Etkin NL, Ticktin $T$ (2010) Advancing an ethno-ecological perspective that integrates theory and method in ethnobotany. In Albuquerque UP, Hanazaki N (eds.) Recent developments and case studies in Ethnobotany. SBEE/NUPEEA, Recife, pp. 33-57.

49. Falé PL, Ferreira C, Rodrigues AM, Frazão FN, Serralheiro ML (2014) Studies on the molecular mechanism of cholesterol reduction by Fraxinus angustifolia, Peumus boldus, Cynaracardunculus and Pterospartum tridentatum infusions. J Med Plant Res 8(1):9-17.

50. Ferreira JM, Sousa DF, Dantas MB, Fonseca SG, Menezes DB, Martins AM, de Queiroz MG (2013) Effects of Bixa orellana L. seeds on hyperlipidemia. Phytother Res 27(1):144-147.

51. Furgione A, Sánchez D, Scott G, Luti Y, Arraiz N, Bermúdez V, Velasco M (2009) Dislipidemias primarias como factor de riesgo para la enfermedad coronaria. Rev Latinoam Hipert 4(1):18-25.

52. Gao Q, Qin WS, Jia ZH, Zheng JM, Zeng CH, Li LS, Liu ZH (2010) Rhein improves renal lesion and ameliorates dyslipidemia in $\mathrm{db} /$ db mice with diabetic nephropathy. Planta Med 76(1):27-33.

53. García-Lazo G, Lauzant-Diaz E, Díaz Batista A, GarcíaMesa M (2015) Plants considered useful for hypoglycemic, antihypertensive or hypolipidemic treatments by patients with peripheral vascular diseases. Rev Cubana Plant Med 20(1):38-47.

54. García Mesa M (2014) Hypolipidemic potential of plants used in Cuba. Pharmacologyonline 1:73-80.

55. Garmendia F, Pando R, Ronceros G (2011) Efecto del aceite de sacha inchi (Plukenetia volubilis $L$.) sobre el perfil lipídico en pacientes con hiperlipoproteinemia. Rev Peru Med Exp Salud Pública 28(4):628-632.

56. Geeraert B, Crombé $F$, HulsmansM, Benhabilès N, Geuns JM, HolvoetP (2010) Stevioside inhibits atherosclerosis by improving insulin signaling and antioxidant defense in obese insulinresistant mice. Int J Obesity 34:569-577.
57. Ghasi S, Nwobodo E, Ofili JO (2000) Hypocholesterolemic effects of crude extract of leaf of Moringa oleifera in high-fat diet fed wistar rats. J Ethnopharmacol 69(1):21-25.

58. Granato EM, Granato MM, Gerenutti M, Glauzer Silva M, Onishi Ferraz H, Duarte Carvalho Vila M (2013) Prospecção fitoquimica da espécie vegetal Trixis antimenorrhoea (Schrank) Kuntze. Rev Bras Farm 94(2):130-135.

59. Habib NC, Honoré SM, Genta SB, Sánchez SS (2011) Hypolipidemic effect of Smallanthus sonchifolius roots on diabetic rats. Biochemical approach. Chem Biol Interact194(1):31-39.

60. Han HY, Yoon SJ, Kim GH (2009) Effects of Compositae plants on plasma glucose and lipid level in streptozotocin induced diabetic rats. J Korean Soc Food Sci Nutr 38(6):674-682.

61. He X, Wang J, Li M, Hao D, Yang Y, Zhang C, He R, Tao R (2014) Eucommia ulmoides Oliv.: ethnopharmacology, phytochemistry and pharmacology of an important traditional Chinese medicine. J Ethnopharmacol 151(1):78-92.

62. Hossain MS, Ahmed M, Islam A (2010) Hypolipidemic and hepatoprotective effects of different fractions of ethanolic extract of inmature leaves of Mangifera indica $L$. in alloxan induced diabetic rats. Int J Pharm Sci Res 1(11):132-138.

63. Hossain MS, Badrul Alam M, Asadujjaman M, Monirul Islam M, Azizur Rahman M, Islam M, Islam A (2011) Antihyperglycemic and antihyperlipidemic effects of different fractions of Stevia rebaudiana leaves in alloxan induced diabetic rats. Int J Pharm Sci Res 2(7):1722-1729.

64. Hurrell JA (2014) Urban Ethnobotany in Argentina: Theoretical advances and methodological strategies. Ethnobiology and Conservation 3: 1-11. doi: 10.15451/ec2014-6-3.3-1-11.

65. Hurrell JA, Pochettino ML (2014) Urban Ethnobotany: theoretical and methodological contributions. In Albuquerque UP, Cruz da Cunha LV, Lucena RP, Alves RN (eds.) Methods and Techniques in Ethnobiology and Ethnoecology.Springer-Humana Press, New York, pp. 293-310.

66. Hurrell JA, Puentes JP (2013) Medicinal and aromatic species of Asteraceae commercialized in the conurbation Buenos Aires-La Plata (Argentina). Ethnobiology and Conservation 2: 1-40. http:// ethnobioconservation.com/index.php/ebc.

67. Hurrell JA, Pochettino ML, Puentes JP, Arenas PM (2013) Del marco tradicional al escenario urbano: Plantas ancestrales devenidas suplementos dietéticos en la conurbación Buenos Aires-La Plata, Argentina. Bol Latinoam Caribe Plant Med Aromat 12(5): 499-515.

68. Hurrell JA, Arenas PM, Cristina I (2015) El conocimiento botánico en zonas urbanas: potenciadores cognitivos comercializados en el área metropolitana de Buenos Aires, Argentina. Gaia Scientia (in press).

69. Husain GM. Chatterjee SS, Singh PN, Kumar V (2011) Hypolipidemic and antiobesity-like activity of standardised extract ofHypericum perforatumL. in rats. Pharmacology 2011:505247, doi:http://dx.doi.org/10.5402/2011/505247.

70. Huseini HF, Larijani B, Heshmat R, Fakhrzadeh H, Radjabipour B, Toliat T. Raza M (2006) The efficacy of Silybum marianum (L.) Gaertn. (silymarin) in the treatment of type II diabetes: a randomized, double-blind, placebo-controlled, clinical trial. Phytother Res 20(12):1036-1039.

71. Hussin M, Hamid AA, Mohamad S, Saari N, Bakar F, Dek SP (2009) Modulation of lipid metabolism by Centella asiatica in oxidative stress rats. J Food Sci 74(2):H72-H78. 
72. Ibrahim SRM, Mohamed GA, Banjar ZM, Kamal HKM (2013) Natural antihyperlipidemic agents: current status and future perspectives. Phytopharmacol 4(3):492-531.

73. Ineedi S, Kumar V (2009) Anti-hyperlipidemic activity of Hyperforin: an in vivo study in rats. Pharmacologyonline 2:133139.

74. Jiang QR (2011) Effects of Lycium barbarum polysaccharides on blood lipid and oxidative stress of aorta in hyperlipidemia rats. Ningxia Medical University, Yinchuan.

75. Johari NZ, Ismail IS, Sulaiman MR, Abas F, Shaari K (2015) Acute toxicity and metabolomics analysis of hypocholesterolemic effect of Mentha piperita aqueous extract in Wistar rats. Int $\mathrm{J}$ Appl Res Nat Prod 8(1):1-11.

76. Jukanti AK, Gau PM, Gowda CLL, Chibbar RN (2012) Nutritional quality and health benefits of chickpea (Cicer arietinum L.): a review. Br J Nutr 108(S1): S11-S26.

77. Jung CH, Jang SJ, Ahn J, Gwon SY, Jeon TI, Kim TW, Ha TY (2012) Alpinia officinarum inhibits adipocyte differentiation and high-fat diet-induced obesity in mice through regulation of adipogenesis and lipogenesis. J Med Food 15(11):959-967.

78. Kamiya K, Tanaka $Y$, Endang H, Umar M, Satake $T$ (2004) Chemical constituents of Morinda citrifolia fruits inhibit copperinduced low-density lipoprotein oxidation. J Agric Food Chem 52(19):5843-5848.

79. Karimi I, Hayatgheybi H, Razmjo M, Yousefi M, Dadyan A (2010) Anti-hyperlipidaemic effects of an essential oil of Melissa officinalis $L$. in cholesterol-fed rabbits. J Appl Biol Sci 4(1):17-22.

80. Kawase A, Yamada A, Gamou Y, Tahara C, Takeshita F, Murata K, Matsuda H, Samukawa K, Iwaki M (2014) Effects of ginsenosides on the expression of cytochrome P450s and transporters involved in cholesterol metabolism. J Nat Med 68(2):395-401.

81. Khaleel AE, Gad MZ, El-Marafhy SA, Hifnawy MS, Abdel-Sattar $E$ (2005) Study of hypocholesterolemic and antiatherosclerotic properties of Medicago sativa L. cultivated in Egypt. J Food Drug Analysis 13(3):212-218.

82. Khanna AK, Rizvi F, Chander R (2002) Lipid lowering activity of Phyllanthus nirurin hyperlipemic rats.J Ethnopharmacol 82(1):19-22.

83. Kiage-Mokua BN, Roos N, Schrezenmeir J (2012) Lapacho tea (Tabebuia impetiginosa) extract inhibits pancreatic lipase and delays postprandial triglyceride increase in rats. Phytother Res 26(12):1878-1883.

84. Kim H, Bartley GE, Rimando AM, Yokoyama W (2010) Hepatic gene expression related to lower plasma cholesterol in hamsters fed high-fat diets supplemented with blueberry peels and peel extract. J Agric Food Chem 58(7):3984-3991.

85. Kocsis I, Hagymási K, Kéry A, Szôke E, Blázovics A (2003) Effects of chicory on pancreas status of rats in experimental dyslipidemia. Acta Biol Szeged 47(1-4):143-146.

86. Koscielny D, Klüßendorf D, Latza R, Schmitt R, Radtke H, Siegel G, Kiesewetter H (1999) The antiatherosclerotic effect of Allium sativum. Atherosclerosis 144(1):237-249.

87. Koshy AS, Anila L, Vijayalakschmi NR (2001) Flavonoids from Garcinia cambogia lower lipid levels in hypercholesterolemic rats. Food Chem 72(3):289-294.

88. Krecman V, Skottova N, Walterova D, Ulrichova J, Simanek V (1998) Silymarin inhibits the development of diet-induced hypercholesterolemia in rats. Planta Med 64(2):138-142.
89. Kumar P, Bhandari U (2013) Protective effect of Trigonella foenumgraecum on monosodium glutamate-induced dyslipidemia and oxidative stress in rats. Indian J Pharmacol 45(2):136-140.

90. Langley PC, Pergolizzi JV, Taylor R, Ridgway C (2015) Antioxidant and associated capacities of Camu-camu (Myrciaria dubia).A systematic review. J Altern Complement Med 21(1):8-14.

91. Lastra Valdés H, Piquet García R (1999) Calendula officinalis. Rev Cubana Farm 33(3):188-194.

92. Lee LS, Cho CW, Hong HD, Lee YC, Choi UK, Kim YC (2013) Hypolipidemic and antioxidant properties of phenolic compound-rich extracts from white ginseng (Panax ginseng) in cholesterol-fed rabbits. Molecules 18(10):12548-12560.

93. Lee SY, Park SL, Hwang JT, Yi SH, Nam YD, Lim SI (2012) Antidiabetic effect of Morinda citrifolia (noni) fermented by Cheonggukjang in $\mathrm{KK}-\mathrm{A}(\mathrm{y})$ diabetic mice. Evid Based Complement Alternat Med 2012:163280, doi: 10.1155/2012/163280.

94. Li J, Yu L, Li N, Wang H (2000) Astragalus mongholicus and Angelica sinensis compound alleviates nephritic hyperlipidemia in rats. Chin Med J 113:310-314.

95. Li MH, Li L, Yang YM, Zhang N, Song XL, Xiao PG (2010) Genus Gentianella Moench: A phytochemical and ethnopharmacological review. Chin Herb Med 2(4):262-271.

96. Liang Y, Chen J, Zuo Y, Ma KY, Jiang Y, Huang Y, Chen ZY (2013) Blueberry anthocyanins at doses of 0.5 and $1 \%$ lowered plasma cholesterol by increasing fecal excretion of acidic and neutral sterols in hamsters fed a cholesterol-enriched diet. Eur J Nutr 52(3):869-975.

97. Lima Portella R, Barcelos RP, da Rosa EJ, Ribeiro EE, da Cruz IB, Suleiman L, Soares FA (2013) Guaraná (Paullinia cupana) effects on LDL oxidation in elderly people: an in vitro and in vivo study. Lipids Health Dis 12:12, doi: 10.1186/1476-511X-12-12.

98. Lin YL, Chou CH, Yang DJ, Chen JW, Tzang BS, Chen YC (2012) Hypolipidemic and antioxidative effects of noni (Morinda citrifolia L.) juice on high- fat/cholesterol-dietary hamsters. Plant Foods Hum Nutr 67(3):294-302.

99. Lino CS, Diógenes JPL, Pereira BA, Faria RA, Andrade Neto M, Alves RS, Queiroz MGR, Sousa FCF, Viana GSB (2004) Antidiabetic activity of Bauhinia forficata extracts in alloxan-diabetic rats. Biol Pharm Bull 27(1)125-127.

100. Littleton RM, Haworth KJ, Tang H, Setchell KD, Nelson S, Hove JR (2013) Automated in vivo platform for the discovery of functional food treatments of hypercholesterolemia. PLoS One 8(1):e52409, doi: 10.1371/journal.pone.0052409.

101. Liu L, Gou XM (2010) Extraction of total saponins from Calendula officinalis $L$. and its effects of anti-oxidation and hypolipidemic. J Mount Agric Biol 3:246-248.

102. Luo Q, Cai Y, Yan Y, Sun M, Corke H (2004) Hypoglycemic and hypolipidemic effects and antioxidant activity of fruit extracts from Lycium barbarum. Life Sci 76:137-149.

103. Ma J, Qiao Z, Xiang X (2011) Aqueous extract of Astragalus mongholicus ameliorates high cholesterol diet induced oxidative injury in experimental rats models. J Med Plants Res 5(5):855-858.

104. Mandukhail SU, Aziz N, Gilani AH (2010) Studies on antidyslipidemic effects of Morinda citrifolia (noni) fruit, leaves and root extracts. Lipids Health Dis 9:88, doi: 10.1186/1476-511X9-88.

105. Mani Badal R, Badal D, Badal P, Khare A, Shrivastava J and Kumar V (2011) Pharmacological action of Mentha piperita on 
lipid profile in fructose-fed rats. Iranian J Pharm Res 10(4):843848.

106. Marchesi M, Parolini C, Diani E, Rigamonti E, Cornelli L, Arnoldi A (2008) Hypolipidaemic and antiatherosclerotic effects of lupin proteins in a rabbit model. $\mathrm{Br} \mathrm{J}$ Nutr 4:1-4.

107. Martin GJ (1995) Ethnobotany. A methods manual.Chapman \& Hall, London.

108. Mateos-Aparicio I, Redondo Cuenca A, Villanueva-Suárez MJ, Zapata-Revilla MA (2008) Soybean, a promising health source. Nutr Hosp 23(4):305-312.

109. Maurya SK, Raj R, Srivastava AK (2009) Anti-dyslipidaemic activity of Glycyrrhiza glabra in high fructose diet induced dyslipidaemic Syrian golden hamsters. Indian J Clin Biochem 24(4)404-409.

110. Maza Cave MP, Díaz Corvalán J, Gómez Lagos R, Maiz Gurruchaga A (2000) Dislipidemias. Ministerio de Salud, Santiago de Chile.

111. Mbikay M (2012) Therapeutic potential of Moringa oleifera leaves in chronic hyperglycemia and dyslipidemia. A review. Front Pharmacol. 3:24, doi:10.3389/fphar.2012.00024.

112. Mchedlishvili D, Kuchukashvili Z, Tabatadze T, Davitaia G (2005) Influence of flavonoids isolated from Satureja hortensis $L$. on hypercholesterolemic rabbits. Indian J Pharmacol 37:259-260.

113. McKay DL, Blumberg JB (2006) A review of the bioactivity and potential health benefits of chamomile tea (Matricaria recutita L.). Phytother Res 20(7):519-530.

114. Megraj KVK, Raju K, Balaraman R, Meenakshisundaram K (2011) Biological activities of some Indian medicinal plants. J Adv Pharm Educ Res 1:12-44.

115. Mesa MD, Ramírez-Tortosa MC, Aguilera CM, Ramírez-Boscá A, Gil A (2000) Efectos farmacológicos y nutricionales de los extractos de Curcuma longa $L$. y de los cucuminoides. Ars Pharmaceutica 41(3):307-321.

116. Miranda Velásquez LG (2010) Actividad hipocolesterolémica de plantas de uso etnobotánico en México. Universidad Autónoma de Nuevo León, Monterrey.

117. Miura $T$, Itoh $Y$, Ishida $T$ (2004) Hypoglycaemic and hypolipidemic activity of the leaf of Smallanthus sonchifolius in genetically type 2 diabetic mice. J Tradit Med 21:275-277.

118. Momtaz S, Abdollahi M (2008) A systematic review of the biological activities of Satureja L. species. Pharmacologyonline 2:34-54.

119. Muir AD, Westcott ND, Prasad K (1999) Extraction, purification and animal model testing of an anti-atherosclerotic lignan secoisolariciresinol diglucoside from flaxseed (Linum usitatissimum). Acta Horticulturae 501:245-248.

120. Muruganandan S, Srinivasan K, Gupta S, Gupta P, Lal J (2005) Effect of mangiferin on hyperglycemia and atherogenicity in streptozotocin diabetic rats. J Ethnopharmacol 97(3):497-501.

121. Mustafa KG, Ganai BA, Akbar S, Dar MY, Masood A (2012) Q-Cell protective efficacy, hypoglycemic and hypolipidemic effects of extracts of Achillea millifolium in diabetic rats. Chin J Nat Med 10(3):185-189.

122. Nada SA, Bashandy SAE, Negm SA (1997) Evaluation of the hypoglycemic activity of a traditional herbal preparation in male diabetic rats. Fitoterapia 68(3):240-244.

123. Nascimento 0, Boleti A, Yuyama L, Lima E (2013) Effects of diet supplementation with Camu-camu (Myrciaria dubia) fruit in a rat model of diet-induced obesity. An Acad Bras Cienc 85(1):55-363.
124. Nayak BS, Marshall JR, Isitor G, Adogwa A (2011) Hypoglycemic and hepatoprotective activity of fermented fruit Juice of Morinda citrifolia (noni) in diabetic rats. Evid Based Complement Alternat Med 2011:875293, doi: http://dx.doi.org/10.1155/2011/875293.

125. Nirmala A, Eliza J, Rajalakshmi M, Priya E, Daisy P (2008) Effect of hexane extract of Cassia fistula barks on blood glucose and lipid profile in streptozotocin diabetic rats. Int $\mathrm{J}$ Pharmacol 4(4):292-296.

126. Nku CO, Ikpi DE, Nna VU, Agiande GU (2014) Altered serum lipid profile in albino Wistar rats following the consumption of Cola nitida rubra (Kola nut). Austr J Basic \& Appl Sci 8(13):82-89.

127. Oré Sifuentes MR (2008) Efectos hipolipémico y antioxidante de Lepidium meyenii en ratas. Universidad Nacional Mayor de San Marcos, Lima.

128. Orekhov AN (2013) Direct anti-atherosclerotic therapy. Development of natural anti-atherosclerotic drugs preventing cellular cholesterol retention.Curr Pharm Design 19(33):59095928.

129. Orrego R, Leiva $E$, Cheel J (2009) Inhibitory effect of three C-glycosylflavonoids from Cymbopogon citratus (Lemongrass) on human low density lipoprotein oxidation. Molecules 14(10):3906-3913.

130. Oulmouden F, Ghalim N, El Morhit M, Benomar H, Daoudi EM, Amrani S (2014) Hypolipidemic and anti-atherogenic effect of methanol extract of fennel (Foeniculum vulgare) in hypercholesterolemic mice. Int J Sci Knowl 3(1):42-52.

131. Pan S, Yu Q, Zhang Y, Wang X, Sun N, Yu Z, Ko K (2012) Dietary Fructus Schisandrae extracts and fenofibrate regulate the serum/hepatic lipid-profile in normal and hypercholesterolemic mice, with attention to hepatotoxicity. Lipids Health Dis 11:120, doi: 10.1186/1476-511X-11-120.

132. Panossian A, Wikman G (2008) Pharmacology of Schisandra chinensis: an overview of Russian research and uses in medicine. J Ethnopharmacol 118(2):183-212.

133. Parab R, Mengi S (2002) Hypolipidemic activity of Acorus calamus $L$. in rats. Fitoterapia 73:451-455.

134. Park SH, Sung YY, Nho KJ, Kim HK (2014) Anti-atherosclerotic effects of Polygonumaviculare L. ethanol extract in ApoE knockout mice fed a Western diet mediated via the MAPK pathway. J Ethnopharmacol 151(3):1109-1115.

135. Paula H, Pedrosa ML, Rossoni Júnior J, Haraguchi FK, Santos C, Silva ME (2009) Effect of an aqueous extract of annatto (Bixa orellana) seeds on lipid profile and biochemical markers of renal and hepatic function in hipercholesterolemic rats. Braz Arch Biol Technol 52(6):1373-1378.

136. Pingale SS (2008) Evaluation of effect of Centella asiatica on CCL4 induced rat liver damage. Pharmacologyonline 3:537-543.

137. Pizziolo VR, Brasileiro BG, Oliveira TT, Nagem TJ (2011) Plantas com possível atividade hipolipidêmica: uma revisão bibliográfica de livros editados no Brasil entre 1998 e 2008. Rev Bras PI Med (Botucatu) 13(1):98-109.

138. Pochettino ML, Hurrell JA (2013) Saberes y plantas en las diagonales: transmisión del conocimiento botánico urbano. Bol Soc Argent Bot 48(Supl):16.

139. Pochettino ML, Puentes JP, Buet Costantino F, Arenas PM, Ulibarri EA, Hurrell JA (2012) Functional foods and nutraceuticals in a market of Bolivian immigrants in Buenos Aires (Argentina). Evid-Based Complement Altern Med 2012:320193, doi: $10.1155 / 2012 / 320193$. 
140. Poon TYC, Ong KL, Yung BM (2011) Review of the effects of the traditional Chinese medicine Rehmannia six formula on diabetes mellitus and its complications. $J$ Diabetes 3:184-200.

141. Potawale SE, Mehta UK, Sadiq MW, Dhalawat HJ, Luniya KP, Mantri RA, Vetal YD (2008) Phytopharmacology of Uncaria tomentosa. A review. Pharmacologyonline 2:197-214.

142. Puentes JP, Hurrell JA (2015) Plantas andinas y sus productos comercializados con fines medicinales y alimentarios en el Área Metropolitana Buenos Aires-La Plata, Argentina. Bol Latinoam Caribe Plant Med Aromat 14(3):206-236.

143. Quinlan $\mathrm{M}(2005)$ Considerations for collecting freelists in the field: examples from Ethnobotany. Field Methods 17(3):1-16.

144. Rafraf M, Zemestani M, Asghari-Jafarabadi M (2015) Effectiveness of chamomile tea on glycemic control and serum lipid profile in patients with type 2 diabetes. J Endocrinol Inv 38(2):163-170.

145. Radjabian T, Fallah Huseini H (2010) Anti-hyperlipidemic and anti-atherosclerotic activities of silymarins from cultivated and wild plants of Silybum marianum L. with different content of flavonolignans. Iranian J Pharm Ther 9(2):63-67.

146. Robert ML, Matthew M, Jay RH (2012) Whole plant based treatment of hypercholesterolemia with Crataegus laevigatain a zebrafish model. BCM Complement Alternat Med 12:105, doi: 10.1186/1472-6882-12-105

147. Roghani $M$, Baluchnejad-Mojarad T, Roghani-Dehkordi $F$ (2005) Hypoglycemic and hypolipidemic effect of chronic oral administration of aerial part of Marrubium vulgare in diabetic rats. J Gorgan Univ Med Sci 7(2):1-5.

148. Roghani M, Aghaie M (2007) The effect Allium ampeloprasum feeding on serum level of glucose, triglyceride, and total cholesterol of diabetic rats. Koomesh J Semnan Med Sci Univ 2(8):73-77.

149. Santanam N, Penumetcha M, Speisky H, Parthasarathy S (2004) A novel alkaloid antioxidant, Boldine and synthetic antioxidant, reduced form of RU486, inhibit the oxidation of $L D L$ in-vitro and atherosclerosis in vivo in $\mathrm{LDLR}^{-/-}$mice. Atherosclerosis 173(2):203-210.

150. Saravanan M, Ignacimuthu S (2015) Hypocholesterolemic effect of Indian medicinal plants. A review. Med Chem 5:1, doi: http://dx.doi.org/10.4172/2161-0444.1000241.

151. Savita S, Sheela K, Sunanda S, Shankar A, Ramakrishna $P_{t}$ Sakey S (2004) Health implicationsof Stevia rebaudiana. J Hum Ecol 15(3):191-194.

152. Semwal RB, Semwal DK, Vermaak I and Viljoen A (2015) A comprehensive scientific overview of Garcinia cambogia. Fitoterapia 102:134-148.

153. Sewani-Rusike CR, Jumbam DN, Chinhoyi LR, Nkeh-Chungag BN (2015) Investigation of hypogycemic and hypolipidemic effects of an aqueous extract of Lupinus albus legume seed in Streptozotocin-induced type I diabetic rats. Afr J Tradit Complement Altern Med 12(2):36-42.

154. Shabrova EV, Tarnopolsky 0, Singh AP, Plutzky J, Vorsa N, Quadro $L$ (2011) Insights into the molecular mechanisms of the antiatherogenic actions of flavonoids in normal and obese mice. PLoS One 6(10):e24634, doi: 10.1371/journal.pone.0024634.

155. Shafi S, Tabassum N (2013) Survey on anti-diabetic plants in Kashmir (India). J AdvPharm Edu Res 3(4):306-318.

156. Shi Y, Guo R, Wang X, Yuan D, Zhang S, Wang J, Yan X, Wang $C$ (2014) The regulation of alfalfa saponin extract on key genes involved in hepaticcholesterolmetabolism in hyperlipidemic rats. PLoS One 9(2):e88282, doi: 10.1371/journal.pone.0088282.

157. Shin JE, Joo Han M, Kim DH (2003) 3-Methylethergalangin isolated from Alpinia officinarum inhibits pancreatic lipase. Biol Pharm Bull 26(6):854-857.

158. Sierra L, Roco J, Alarcón G, Medina M, van Nieuwenhove, Peral de Bruno M, Jerez S (2015) Dietary intervention with Salvia hispanica (Chia) oil improves vascular function in rabbits under hypercholesterolaemic conditions. J Funct Foods 14:641-649.

159. Singh PK, Baxi DB, Mukherjee R, Ramachandran AV (2010) Evaluation on the efficacy of a poly herbal supplement along with exercise in alleviating dyslipidemia, oxidative stress and hepatic and renal toxicity associated with Type-1 diabetes. J Herbal Med Toxicol 4(1):207-215.

160. Singh S, Garg V (2014) Antidiabetic, antidyslipidymic and antioxidative potential of methanolic root extractofStevia rebaudiana(Bertoni) on alloxan induced diabetic mice. World $\mathrm{J}$ Pharm Pharmaceut Sci 3(7):1859-1872.

161. Solà R, Godàs G, Ribalta J, Vallvé JC, Girona J, Anguera A, Ostos M, Recalde D, Salazar J, Caslake M, Martín-Luján F, SalasSalvadó J, Masana L (2007) Effects of soluble fiber (Plantago ovata husk) on plasma lipids, lipoproteins, and apolipoproteins in men with ischemic heart disease. Am J Clin Nutr 85(4):11571163.

162. Solà R, Bruckert E, Valls RM, Narejos $S$, Luque $X$, Castro-Cabezas M, Doménech G, Torres F, Heras M, Farrés X, Vaquer JV, Martínez JM, Almaraz MC, Anguera A (2010) Soluble fibre (Plantago ovata husk) reduces plasma low-density lipoprotein (LDL) cholesterol, triglycerides, insulin, oxidised LDL and systolic blood pressure in hypercholesterolaemic patients: A randomised trial. Atherosclerosis 211(2):630-637.

163. Souza MO, Silva M, Silva ME, Oliveira RP, Pedrosa ML (2010) Diet supplementation with açaí (Euterpe oleracea) pulp improves biomarkers of oxidative stress and the serum lipid profile in rats. Nutrition 26:804-810.

164. Souza M0, Souza E Silva L, de Brito Magalhães CL, de Figueiredo BB, Costa DC, Silva ME, Pedrosa ML (2012) The hypocholesterolemic activity of açaí (Euterpe oleracea Mart.) is mediated by the enhanced expression of the ATP-binding cassette, subfamily $\mathrm{G}$ transporters 5 and 8 and low-density lipoprotein receptor genes in the rat. Nutr Res 32(12):976-984.

165. Souza SP, Pereira LLS, Souza AA, dos Santos CD (2011) Inhibition of pancreatic lipase by extracts of Baccharis trimera (Less.) DC., Asteraceae. Evaluation of antinutrients and effect on glycosidases. Rev Bras Farmacogn 21(3):450-455

166. Souza SP, Pereira LLS, Souza AA, Santos CD (2012) Seleção de extratos brutos de plantas comatividade antiobesidade. Rev Bras Plantas Med 14(4):643-648.

167. Stepp JR (2005) Advances in ethnobiological field methods. Field Methods 17(3):211-218.

168. Sung YY, Yoon T, Yang WK, Kim SJ, Kim DS, Kim HK (2013) The antiobesity effect of Polygonum aviculare $\mathrm{L}$. ethanol extract in high-fat diet-induced obese mice. Evid Based Complement Alternat Med 2013:626397, doi: 10.1155/2013/626397.

169. Susalit E, Agus N, Effendi I, Tjandrawinatac RR, Nofiarnyc D, Perrinjaquet-Moccetti T, Verbruggen M (2011) Olive (Olea europaea) leaf extract effective in patients with stage-1 hypertension: comparison with captopril. Phytomedicine 18:251258.

170. Tomaz Pacheco J, Beltrame Daleprame J, Teles Boaventura $G$ (2011) Impact of dietary flaxseed (Linum usitatissimum) 
supplementation on biochemical profile in healthy rats. Nutr Hosp 26(4):798-802.

171. Valencia EF (2005) Smallanthussonchifoliusen el tratamiento de hiperlipoproteinemias e hipercolesterolemia inducidas en ratas albinas. Rev Fac Med Hum 5(1):27-31.

172. Vasques CA, Rossetto $S$, Halmenschlager $G$, Linden $R$, Heckler E, Fernandez NS, Alonso JL (2008) Evaluation of the pharmacotherapeutic efficacy of Garcinia cambogia plus Amorphophallus konjac for the treatment of obesity. Phytother Res 22(9):1135-1140.

173. Vázquez-Ovando A, Rosado-Rubio G, Chel-Guerrero L, BetancurAncona $D$ (2009) Physicochemical properties of a fibrous fraction from chia (Salvia hispanica L.). Food Sci Technol 42:168-173.

174. Vecera R, Orolin J, Skottová N, Kazdová L, Oliyarnik O, Ulrichová J, Simánek V (2007) The influence of maca (Lepidium meyenii) on antioxidant status, lipid and glucose metabolism in rat. Plant Food Hum Nutr 62(2):59-63.

175. Verma SK, Singh M, Jain P, Bordia A (2004) Protective effect of ginger, Zingiber officinale on experimental atherosclerosis in rabbits. Indian J Exp Biol 42(7):736-738.

176. Vignale ND, Gurni AA (2007) Aplicaciones de la micrografía en la identificación de especies. Actas I Simp Int Invest. Universidad Católica de Santiago del Estero. Departamento Académico de San Salvador (UCSE-DASS). San Salvador de Jujuy, pp. 433-444.

177. Viveros A, Centeno C, Arija I, Brenes A (2007) Cholesterollowering effects of dietary lupin (Lupinus albus) in chicken diets. Poult Sci 86(12):2631-2638.

178. Wang D, Zhuang Y, Tian Y, Thomas GN, Ying MZ, Tomlinson NB (2012) Study of the effects of total flavonoids of Astragalus on atherosclerosis formation and potential mechanisms. Oxid Med Cell Long 2012:282383, doi: http://dx.doi.org/10.1155/2012/282383.

179. Wang K, Cao P, Shui WZ, Yang QX, Tang Z, Zhang Y (2015) Angelica sinensis polysaccharide regulates glucose and lipid metabolism disorder in prediabetic and streptozotocin-induced diabetic mice through the elevation of glycogen levels and reduction of inflammatory factors. Food Funct 6:902-909.

180. Wärnberg J, Marcos A, Bueno G, Moreno LA (2009) Functional benefits of psyllium fiber supplementation. Curr Top Nutraceut Res 7(2):55-64.

181. Wei JM, Wang X, Gong H, Shi YJ, Zou Y (2013) Ginkgo suppresses atherosclerosis through downregulating the expression of connexin 43in rabbits. Arch Med Sci 9(2):340-346.

182. Weidner C, Wowro SJ, Freiwald A, Kodelja V, Abdel-Aziz H, Kelber 0, Sauer S (2014) Lemon balm extract causes potent antihyperglycemic and antihyperlipidemic effects in insulinresistant obese mice. Molec Nutr Food Res 58(4):903-907.

183. Wider B, Pittler MH, Thompson-Coon J, Ernst E (2009) Artichoke leaf extract for treating hypercholesterolaemia.Cochrane Database Syst Rev 7:4, doi: 10.1002/14651858

184. Wu YC, Hsieh CL (2011) Pharmacological effects of Radix Angelica Sinensis (Danggui) on cerebralinfarction. Chin Med 6:32, doi: 10.1186/1749-8546-6-32.

185. Xia DZ, Yu XF, Wang HM, Ren QY, Chen BM (2010) Anti-obesity and hypolipidemic effects of ethanolic extract from Alpinia officinarum Hance (Zingiberaceae) in rats fed high-fat diet. $J$ Med Food 13(4):785-791

186. Xie Z, Liang G, Zhang L, Wang Q, Qu Y, Gao Y, Lin L, Ye S, Zhang J, Wang H, Zhao GP, Zhang $\mathrm{OH}$ (2009) Molecular mechanisms underlying the cholesterol-lowering effect of Ginkgo biloba extract in hepatocytes: a comparative study with lovastatin. Acta Pharmacol Sin 30(9):1262-1275.

187. Yadav UCS, Baquer NZ (2014) Pharmacological effects of Trigonella foenum-graecum $\mathrm{L}$. in health and disease. Pharm Biol 52(2):243-254

188. Yamamoto M, Uemura T, Nakama S, Uemiya M, Kumagai A (1983) Serum HDL-cholesterol-increasing and fatty liver-improving actions of Panax ginseng in high cholesterol diet-fed rats with clinical effect on hyperlipidemia in man. Am J Chin Med 11(14):96-101.

189. Yanai $\mathrm{H}$, Adachi $\mathrm{H}$, Katsuyama H, Hamasaki $\mathrm{H}$ and AkahitoSako A (2015) Anti-atherosclerotic effects of konjac. Funct Foods Health Dis 5(4):136-144

190. Yang MY, Peng CH, Chan KC, Yang YS, Huang CN, Wang CJ (2010) The hypolipidemic effect ofHibiscus sabdariffapolyphenols via inhibiting lipogenesis and promoting hepatic lipid clearance. $\mathrm{J}$ Agric Food Chem 58(2):850-859.

191. Yao P, Song F, Li K, Zhou S, Liu S, Sun X, Nussler AK, Liu L (2007) Ginkgo biloba extract prevents ethanol induced dyslipidemia. An J Chin Med 35(4):643-652

192. Yen GC, Hsieh CL (1998) Antioxidant activity of extracts from Duzhong (Eucommia ulmoides) toward various lipid peroxidation models in vitro.J Agric Food Chem 46(10):3952-3957.

193. Zarei A, Changizi-Ashtiyani S, Rezaei A, Sheidaee H, Nabiyoni F (2014) The effect of Chelidonium majus extract on the lipid profile and activity of pituitary-gonadal axis in hypercholesterolemic rats. Zahedan J Res Med Sci 16(10):18-22.

194. Zarei A, Changizi-Ashtiyani S, Taheri S, Hosseini N (2015) A brief overview of the effects of Melissa officinalis extract on the function of various body organs. Zahedan J Res Med Sci 17(7):2934.

195. Zhong XF, Huang GD, Luo T, Deng ZY, Hu JN (2012) Protective effect of rhein against oxidative stress-related endothelial cell injury. Mol Med Rep 5(5):1261-1266.

196. Zhou L, Shi Y, Guo R, Liang M, Zhu X, Wang C (2014) Digital geneexpression profiling analysis of the cholesterol-lowering effects of alfalfa saponin extract on laying hens. PLoS One 9(6):e98578, doi: 10.1371/journal.pone.0098578.

197. Zhou YX, Xia W, Yue W, Peng C, Rahman K, Zhang H (2015) Rhein: a review of pharmacological activities. Evid Based Complement Alternat Med 2015:578107, doi: 10.1155/2015/578107.

198. Zou YP, Lu YH, Wei DZ (2005) Hypocholesterolemic effects of a flavonoid-rich extract of Hypericum perforatuminratsfed a cholesterol-rich diet.J Agric Food Chem 53(7):2462-2466.

Received: 9 July 2015

Accepted: 5 November 2015

Published: 16 November 2015 